\title{
The TW Hydrae association: trigonometric parallaxes and kinematic analysis ${ }^{\star}$
}

\author{
C. Ducourant ${ }^{1,2,3}$, R. Teixeira ${ }^{3,1}$, P. A. B. Galli ${ }^{3,1}$, J. F. Le Campion ${ }^{1}$, A. Krone-Martins ${ }^{6,3,1}$, B. Zuckerman ${ }^{4}$, \\ G. Chauvin ${ }^{5}$, and I. Song ${ }^{7}$
}

1 Univ. Bordeaux, LAB, UMR 5804, 33270 Floirac, France e-mail: ducourant@obs.u-bordeaux1.fr

2 Observatoire Aquitain des Sciences de l'Univers, CNRS-UMR 5804, BP 89, 33270 Floirac, France

3 Instituto de Astronomia, Geofísica e Ciências Atmosféricas, Universidade de São Paulo, Rua do Matão, 1226 - Cidade Universitária, 05508-900 São Paulo - SP, Brazil

4 Department of Physics \& Astronomy, UCLA, Los Angeles, CA 90095, USA

${ }^{5}$ Laboratoire d'Astrophysique, Observatoire de Grenoble, 414 rue de la piscine, 38400 Saint-Martin d'Hères, France

${ }^{6}$ SIM, Faculdade de Ciências, Universidade de Lisboa, Ed. C8, Campo Grande, 1749-016, Lisboa, Portugal

7 Department of Physics \& Astronomy, the University of Georgia, Athens, GA 30605 USA

Received 13 June 2013 / Accepted 7 January 2014

\section{ABSTRACT}

\begin{abstract}
Context. The nearby TW Hydrae association (TWA) is currently a benchmark for the study of the formation and evolution of young low-mass stars, circumstellar disks, and the imaging detection of planetary companions. For these studies, it is crucial to evaluate the distance to group members in order to access their physical properties. Membership of several stars is strongly debated and age estimates vary from one author to another with doubts about coevality.

Aims. We revisit the kinematic properties of the TWA in light of new trigonometric parallaxes and proper motions to derive the dynamical age of the association and physical parameters of kinematic members.

Methods. Using observations performed with the New Technology Telescope (NTT) from ESO we measured trigonometric parallaxes and proper motions for 13 stars in TWA.

Results. With the convergent point method we identify a co-moving group with 31 TWA stars. We deduce kinematic distances for seven members of the moving group that lack trigonometric parallaxes. A traceback strategy is applied to the stellar space motions of a selection of 16 of the co-moving objects with accurate and reliable data yielding a dynamical age for the association of $t \simeq$ $7.5 \pm 0.7$ Myr. Using our new parallaxes and photometry available in the literature we derive stellar ages and masses from theoretical evolutionary models.

Conclusions. With new parallax and proper motion measurements from this work and current astrometric catalogs we provide an improved and accurate database for TWA stars to be used in kinematical analysis. We conclude that the dynamical age obtained via traceback strategy is consistent with previous age estimates for the TWA, and is also compatible with the average ages derived in the present paper from evolutionary models for pre-main-sequence stars.
\end{abstract}

Key words. parallaxes - proper motions - brown dwarfs - open clusters and associations: individual: TW Hydrae association stars: pre-main sequence

\section{Introduction}

The discovery of nearby young stars, brown dwarfs, and extrasolar planets has grown substantially in the last decade. In this context, the possibility of accurately determining their physical properties has attracted particular interest to the solar neighborhood. Since the discovery of the young $(t \simeq 8 \mathrm{Myr}$, de la Reza et al. 2006), and nearby ( $d \simeq 50$ pc, Zuckerman \& Song 2004) TW Hydrae association (TWA) by Kastner et al. (1997), important progress has been made in the identification of young stars near the Sun, more than 200 of which have been cataloged.

TW Hydrae association is among the closest of the very young associations and for this reason it is a benchmark for the study of stellar and sub-stellar formation and early evolution. The study of the TWA region by Webb et al. (1999) demonstrated the power of the ROSAT All-Sky X-ray survey to reveal

\footnotetext{
* Based on observations performed at the European Southern Observatory, Chile (79.C-0229, 81.C-0143, 82.C-0103, 83.C-0102, 84.C-0014).
}

members not only of TWA but also of other, subsequently identified, youthful nearby associations. A few years later Gizis (2002) identified two free-floating brown dwarfs members of TWA, one of which, 2M1207, soon become famous as the host of the first imaged planet-mass secondary (2M1207b) outside of our solar system (Chauvin et al. 2004). Many TWA members exhibit a signature of dusty disks (e.g., Riaz \& Gizis 2008, 2012; Looper et al. 2010a; Matthews et al. 2007; Schneider et al. 2012a,b). The age of TWA corresponds to the timescale of the end of disk accretion and giant planet building processes. Recent work by Bergin et al. (2013) presents evidence that the disk around TW Hydrae (TWA 1) is still capable of forming a planetary system. The youth and proximity of TWA presents a particularly favorable situation for spatial resolution of disk structures of tens to hundreds of au.

Dynamical measurements of low-mass binaries, for example TWA 22 (Bonnefoy et al. 2009) and TWA 5 (Neuhäuser et al. 2010), may provide unique opportunities for the derivation of individual masses necessary for the calibration of theoretical 
A\&A 563, A121 (2014)

Table 1. Absolute trigonometric parallaxes and proper motions derived in this work for the 13 TWA stars.

\begin{tabular}{|c|c|c|c|c|c|c|c|c|c|}
\hline Star & $\begin{array}{c}\alpha \\
\text { (h:m:s) }\end{array}$ & $\begin{array}{c}\delta \\
\left({ }^{\circ},{ }^{\prime \prime}\right)\end{array}$ & $\begin{array}{c}\pi \\
(\mathrm{mas})\end{array}$ & $\begin{array}{c}d \\
(\mathrm{pc})\end{array}$ & $\begin{array}{l}\mu_{\alpha} \cos \delta \\
(\mathrm{mas} / \mathrm{yr})\end{array}$ & $\begin{array}{c}\mu_{\delta} \\
\text { (mas/yr) }\end{array}$ & $\begin{array}{c}\Delta \pi \\
(\mathrm{mas})\end{array}$ & $\begin{array}{c}\Delta \mu_{\alpha} \cos \delta \\
\text { (mas/yr) }\end{array}$ & $\begin{array}{c}\Delta \mu_{\delta} \\
\text { (mas/yr) }\end{array}$ \\
\hline TWA 1 & 110551.97 & -344216.9 & $20.8 \pm 4.0$ & $48.1 \pm 9.3$ & $-68.4 \pm 1.5$ & $-8.0 \pm 1.5$ & 0.78 & -7.18 & 1.11 \\
\hline TWA 2 & 110913.88 & -300139.7 & $24.0 \pm 4.8$ & $41.6 \pm 8.3$ & $-87.7 \pm 2.3$ & $-7.9 \pm 2.3$ & 0.81 & -7.20 & 0.24 \\
\hline TWA 5 & 113155.46 & -343628.8 & $20.5 \pm 2.4$ & $48.7 \pm 5.7$ & $-75.8 \pm 1.0$ & $-18.3 \pm 1.0$ & 0.73 & -7.00 & 0.28 \\
\hline TWA 7 & 104230.20 & -334016.0 & $29.0 \pm 2.1$ & $34.5 \pm 2.5$ & $-114.4 \pm 0.8$ & $-19.1 \pm 0.8$ & 0.85 & -7.24 & 1.45 \\
\hline TWA $8 A$ & 113241.32 & -265155.6 & $23.4 \pm 2.0$ & $42.8 \pm 3.7$ & $-87.1 \pm 0.8$ & $-28.0 \pm 0.8$ & 0.89 & -7.62 & -1.00 \\
\hline TWA 8B & 113241.23 & -265208.7 & $25.9 \pm 2.0$ & $38.6 \pm 3.0$ & $-86.5 \pm 0.9$ & $-25.0 \pm 0.9$ & 0.89 & -7.62 & -1.00 \\
\hline TWA 9A & 114824.22 & -372849.2 & $19.1 \pm 2.7$ & $52.3 \pm 7.4$ & $-53.1 \pm 3.9$ & $-24.9 \pm 3.9$ & 0.71 & -7.05 & 0.10 \\
\hline TWA 9B & 114823.77 & -372848.3 & $19.2 \pm 1.1$ & $52.1 \pm 3.0$ & $-51.0 \pm 0.6$ & $-18.1 \pm 0.6$ & 0.71 & -7.05 & 0.10 \\
\hline TWA 10 & 123504.31 & -413638.3 & $16.2 \pm 1.0$ & $61.5 \pm 3.8$ & $-64.6 \pm 0.4$ & $-30.3 \pm 0.4$ & 0.5 & -6.58 & -0.52 \\
\hline TWA 12 & 112105.55 & -384516.2 & $15.4 \pm 1.7$ & $65.1 \pm 7.2$ & $-66.2 \pm 0.5$ & $-7.4 \pm 0.5$ & 0.67 & -6.80 & 0.70 \\
\hline TWA 21 & 101314.85 & -523054.1 & $19.8 \pm 1.4$ & $50.4 \pm 3.6$ & $-61.3 \pm 0.6$ & $10.6 \pm 0.6$ & 1.03 & -7.49 & 3.39 \\
\hline TWA 23 & 120727.44 & -324700.0 & $20.6 \pm 1.8$ & $48.4 \pm 4.2$ & $-75.8 \pm 0.9$ & $-25.7 \pm 0.9$ & 0.74 & -7.23 & -0.79 \\
\hline TWA 26 & 113951.21 & -315921.2 & $26.2 \pm 1.1$ & $38.1 \pm 1.6$ & $-93.3 \pm 0.5$ & $-27.5 \pm 0.5$ & 0.64 & -6.30 & 0.11 \\
\hline
\end{tabular}

Notes. For each star we provide its position (epoch $=2000.0$ ), absolute parallax, distance, absolute proper motions, and the $\Delta$ corrections applied to relative values of parallax and proper motions. For example, to obtain absolute parallax for TWA $1, \pi_{\text {relative }}+0.78=\pi$ given in Col. 4 .

evolutionary models. These evolutionary models can also be calibrated by use of kinematic traceback, a technique that was employed by (Ortega et al. 2002) to derive an age of $12 \mathrm{Myr}$ for the $\beta$ Pictoris group. However, other, more recent traceback studies have produced older or indeterminate ages (see summary in Sect. 4 of Binks \& Jeffries (2014). A major goal of the present paper is to improve the accuracy and reliability of the traceback age for the TWA.

Distance from the Earth is a key parameter that enables the physical characterization of objects and kinematical studies of their origin. It is precisely the high quality of astrometric measurements that makes the solar neighborhood, and consequently TWA, a precious laboratory. The association as a whole is also interesting since its kinematics and origin remain unclear. It is located far from molecular clouds and at the near boundary of the Lower Centaurus Crux (LCC) subgroup of the ScorpiusCentaurus (Sco-Cen) association.

Membership and age of TWA stars have been much debated (Song et al. 2003; Ortega et al. 2004; Mamajek 2005; de la Reza et al. 2006; Teixeira et al. 2009; Schneider et al. 2012b; Weinberger et al. 2013). The conclusions of these authors relied heavily on the type and accuracy of the data they used. A rich discussion of the constitution of TWA and a useful source of data utilized by many authors is presented in Mamajek (2005). In his paper, Mamajek collected proper motions from different sources with inhomogeneous quality. The situation for radial velocities was equivalent and trigonometric parallaxes were available for only five Hipparcos stars. An important step in the evaluation of the age of the association was pioneered by Makarov et al. (2005) and another was developed by de la Reza et al. (2006) who applied a traceback strategy to the five HiPPARcos TWA stars. They derived the epoch of minimum volume corresponding to the dynamical age of the association. Since then, the number of identified TWA members has increased as has the quality and availability of data.

In this context, we present here trigonometric parallax and proper motion measurements for 13 TWA stars performed with the ESO/NTT telescope located at La Silla (Chile). The recent increase of TWA stars with measured trigonometric parallaxes (this work and Weinberger et al. 2013), and the publication of the astrometric proper motion catalogs, SPM4 (Girard et al. 2011) and UCAC4 (Zacharias et al. 2013), allows us to set up an extensive and accurate database for TWA stars. The PPMXL catalog
(Roeser et al. 2010) was excluded from the present work since it provides proper motions of much lower internal accuracy than the other two catalogs.

Using a convergent point (CP) analysis (Galli et al. 2012) we identify a group of co-moving stars in TWA. Based on a traceback strategy we derive a core group converging back in time toward a minimum volume in space that corresponds to the dynamical age of the association.

This paper is organized as follows. In Sect. 2 we present the observational material and describe the reduction procedure that leads to the astrometric and photometric properties of our targets. In Sect. 3 we present an updated astrometric database for the 34 proposed members of the association (TWA 1 - TWA 34). Section 4 describes our convergent point analysis and the determination of kinematic parallaxes for group members with unknown trigonometric parallax. We present in Sect. 5 a traceback analysis leading to a convincing traceback age for a core group of the association. Section 6 presents an HR diagram of TWA along with age and mass estimates as derived from stellar evolutionary models. Our conclusions are summarized in Sect. 7.

\section{Data}

For the present work we set up a list of all TWA members without parallax measurements. Excluded were some resolved tight binaries for which an astrometric solution might be problematic and objects with (too) large photometric distances; TWA 1 and TWA 9A, which benefit from a HIPPARcos parallax, were included in our final list as control stars. The list was then reduced to 15 stars during the observations to fit the allocated time. Two stars were observed but are not presented in this paper since a reasonable solution could not be derived. The remaining $13 \mathrm{ob}-$ jects are presented in Table 1 .

\subsection{Observations}

Astrometric and photometric $(V, R, I)$ observations were performed in direct imaging mode with the ESO/NTT telescope. For the astrometric project, nine observational epochs were acquired with a total of 36 half nights spread over almost three years between 2007 and 2010. A set of 3730 exposures were taken, concentrated in 13 directions corresponding to the selected members of TWA for which a parallax measurement was 
required. Observational epochs were required at specific dates to maximize the parallactic factors of most targets. Given that the targets were spread over $3 \mathrm{~h}$ in right ascension, we had to find a compromise between the maximization of parallax factors and the observability of all targets during a night. All observations were realized around transit to minimize the differential color refraction effects (DCR). Multiple exposures taken over three nights were performed at each epoch to average atmospheric effects and to enhance the signal-to-noise ratio $(\mathrm{S} / \mathrm{N})$. For most objects, two exposure times were selected, a short one selected to optimize $\mathrm{S} / \mathrm{N}$ of the bright targets and a longer one for an optimized $\mathrm{S} / \mathrm{N}$ of the faint surrounding stars. We made extreme efforts to observe during transit to minimize the zenith distance and consequently DCR effects that could induce a factious parallax to a target with a significantly different color than its surrounding background stars.

The program started with the SUSI2 instrument (observations in 2007) but unfortunately SUSI2 was decommissioned and the program was transported to the EFOSC2 instrument in 2008. The change of instrument had repercussions on the quality of the observations and the precision goal of the project was degraded by almost a factor of 2 .

Frames were measured using the DAOPHOT II package (Stetson 1987), fitting a stellar point-spread function for each frame. Finally, we created catalogs of measured positions $(x, y)$, internal magnitudes, and associated errors for all objects in each frame.

\subsection{Trigonometric parallax and proper motion determination}

The catalogs that issued from the CCD frames are crosscorrelated and compiled in a meta-catalog containing, for each object in a field, its measurements on each CCD-frame.

A frame, hereafter master frame, is then selected among the various observations to compute equatorial coordinates of each object in the field using 2MASS (Cutri et al. 2003) as a reference catalog. These equatorial coordinates are necessary for the parallactic factors calculation, and for this reason it is convenient to work in a frame oriented in equatorial coordinates. This step is equivalent to scale (size of the pixel) and rotate the master frame to align it on the axes of the 2MASS catalog. The selection of the master frame is a delicate problem since it will define, via the reference catalog used, the reference frame on which each CCD-frame will be projected. It is therefore crucial that it be as free as possible of distortion and that it contain as many stars as possible of various magnitudes so that the classical reduction performed with 2MASS will be as accurate as possible.

All measurements of a field are globally reduced through a block-adjustment-type iterative procedure described in Ducourant et al. (2007, 2008). The philosophy of this treatment is to compute simultaneously the unknown parameters of all stars (correction to standard coordinates, proper motions, parallaxes) and the unknown plate parameters of all frames. The system is overdetermined and it would be, in principle, possible to derive all of the parameters by one inversion.

Nevertheless, the system of normal equations will be of $2 N_{*} N_{\mathrm{F}}$ equations for $5 N_{*}+6 N_{\mathrm{F}}$ unknowns if we consider $N_{\mathrm{F}}$ frames containing $N_{*}$ stars, leading to a matrix of large dimensions (typically a few tens of thousands of equations for thousands of unknowns for a few hundred frames containing 50 to 100 stars). The size of such a large matrix leads the user to prefer an iterative approach to solve the system. We use a Gauss-Seidel method allowing one to first determine the plate constants assuming an a priori value for all stellar parameters. The stellar parameters are determined for each star using modified plate coordinates, the plate constants are re-computed, and so on until convergence which is generally reached after two or three iterations.

This system is ill conditioned which means that the iterative solving of the normal equations will converge toward one of the various solutions of the problem, but not necessarily to the most physical one. To constrain the convergence to the most physical solution, one must add a constraint. Generally one assumes that the sum of parallaxes in the field should be zero (excluding the target from this mean); the same condition applies for proper motions. Convergence is reached after a few iterations and a relative solution (proper motion and parallax) is derived for all stars in the field.

A statistical conversion from relative to absolute parallax and proper motions - based on the Besançon Galaxy model (Robin et al. 2003, 2004) - is applied to derive final estimates of absolute proper motions and trigonometric parallaxes (see Ducourant et al. 2007, for a detailed description).

The procedure described in this section is applied to all 13 TWA stars observed in our campaign. The results of this investigation, including the corrections applied to the relative quantities in order to derive absolute astrometric parameters, are given in Table 1.

\subsection{Astrometric validation}

In the following we compare the parallaxes and proper motions derived in this work with published results in order to evaluate our external errors.

In the case of trigonometric parallaxes, a comparison with HipParcos is difficult, because only five TWA stars are in that catalog. Recently, in a project parallel to the one presented here, Weinberger et al. (2013) radically improved the situation by publishing parallaxes for 14 TWA systems (16 stars). A comparison of Table 1 with the re-reduction of Hipparcos (van Leeuwen 2007) is restricted to only two stars (TWA 1 and TWA 9A), while there are six stars in common with Weinberger et al. (2013). For five of these six the internal errors listed in our Table 1 are larger than the internal errors listed in their Table 2. Although all six parallaxes appear to be consistent at the one-sigma level (Fig. 1), when comparing our results with Weinberger et al. (2013) we notice an apparent systematic offset of unknown origin. The size of the offset, a few mas, is comparable to the mean internal precision (2.2 mas) of the trigonometric parallaxes derived in the present paper.

We compared our proper motions with those from the recent release of the UCAC4 catalog that provides a dense and precise astrometric source of data with an announced precision of 1-10 mas/yr depending on magnitude and observing history. The mean internal precision of the proper motions derived in the present paper is $1.0 \mathrm{mas} / \mathrm{yr}$. Figure 2 shows the comparison of the measured proper motions given in Table 1 with UCAC4 proper motions. We observe a reasonable agreement in both coordinates and no systematic trend. However large discrepancies (beyond three sigmas) can be observed. The origin of these discrepancies is unclear and can be multiple. Binarity is probably one cause. It is also probable that the formal errors in both works are occasionally underestimated. Nevertheless, one must keep in mind that in the present work, the time base of observations is three years, optimized for parallax work but which is rather short for accurate proper motion determination, especially for multiple systems. For this reason, in most cases we used proper motions issued from UCAC4 in our kinematic analysis. 


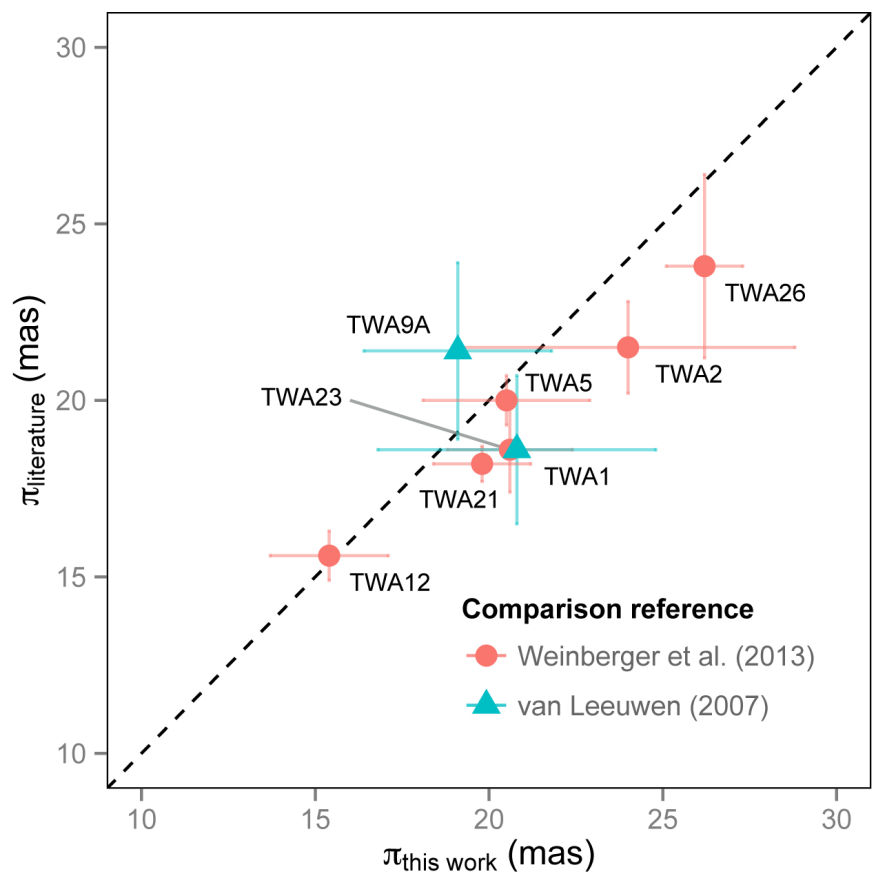

Fig. 1. Comparison of trigonometric parallaxes determined in this work with the published parallaxes given in Hipparcos (van Leeuwen 2007) and Weinberger et al. (2013). The dotted line represents perfect correlation. TWA 23, which was measured by us and by Weinberger et al., sits almost under TWA 1 . The error bar on our measurement is $+/-1.8$ mas.

\subsection{Photometry}

Photometric data were acquired during three consecutive nights (3-5 April 2009) using the Bessel $V, R$ and Gunn $i$ ESO filters (ESO\#641,642,705). We present in Table 2 the photometry derived for the TWA stars observed in this work.

The source TWA 7 has a $V$ magnitude that does not agree with Tycho-2 (Høg et al. 2000), but is in perfect agreement with the accurate photometric variability project of Messina et al. (2010). It is likely that the faint object close to TWA $7\left(\delta \sim 2.4^{\prime \prime}\right.$; Messina et al. 2010) was included in the Tycho-2 photometry. Our $V$ and $I$ magnitudes for TWA 8B do not agree with those published by Messina et al. (2010). Exposure times in our work were optimized for the primary TWA $8 \mathrm{~A}$ which is much brighter; this may explain the poor results obtained here for the secondary component. For TWA 9A and TWA 9B no reliable photometry could be obtained here.

\section{An updated astrometric database for TWA}

Using the parallaxes and proper motions derived in this work, the recent results of Weinberger et al. (2013), and the new release of SPM4 and UCAC4, we set up an updated astrometric database for TWA stars that allows us to revisit completely the kinematics of the association. The selection of data used in this analysis is a very important step, because published values can vary widely from one source to another. In the following we discuss our criteria for choosing (among various sources) parallaxes, proper motions, and radial velocities for TWA stars.

We use the trigonometric parallaxes from the new reduction of Hipparcos (van Leeuwen 2007) when available and combine them with the associated Tycho- 2 proper motions. For the remaining TWA stars we use the trigonometric parallaxes derived in this work which we supplement with those provided by
Weinberger et al. (2013). The average parallax error is 1.5 mas (Table 3 ) yielding an average relative error of $8 \%$.

In the case of proper motions, we searched the SPM4 and UCAC4 catalogs. For common stars in both catalogs we favored the one with the lowest errors in proper motions which in general corresponds to UCAC4. In the case of discrepant values we compared the proper motion values with other sources (e.g., this work and Weinberger et al. 2013) to decide between the two catalogs. For the specific cases of multiple systems that exhibit a poor or inconsistent solution (e.g., TWA 13 and TWA 15) and stars lacking information in global catalogs, we adopted the proper motions from small field astrometry (this work and Weinberger et al. 2013) when no other reliable data were available. Doing so, the average error in proper motions is $1.8 \mathrm{mas} / \mathrm{yr}$ and $1.6 \mathrm{mas} / \mathrm{yr}$, respectively, in right ascension and declination. This yields an average relative error in proper motions better than $4 \%$.

A proper motion for TWA 29 of $(-89.4,-20.9) \pm$ $(10,10) \mathrm{mas} / \mathrm{yr}$, was estimated by Schneider et al. (2012a) based on 2MASS and WISE positions. However, the proper motion derived by Looper et al. (2007) from UKST observations combined with 2 MASS and DENIS positions is radically different $(-220,+20) \pm(70,100) \mathrm{mas} / \mathrm{yr}$, so we decided to remeasure this proper motion using the ESO archive database (15 NTT/SOFI NIR images taken from 2003 to 2006) and the WISE ( 2010), 2MASS $(\sim 2000)$, DENIS( $\sim 1999)$, and GSC2( 1984) published positions. We derived a proper motion of $(-71,-23) \pm(7,3)$ mas/yr. This object has been included in our analysis despite the large error-bars.

For radial velocities we collected the available measurements in the literature and we rarely had to perform a selection between the various sources, because they exist in small number compared to sources for proper motions. In the case of TWA 4, a tight quadruple system (Messina et al. 2010), values found in the literature refer either to the A or B component while here we are using a Tycho- 2 proper motion for this star that refers to the photo-center of the system. We therefore adopted the radial velocity of the center of mass of the system provided by Torres et al. (2003). We present the results of our data compilation for TWA stars in Table 3. In the following sections we investigate the kinematics of the association and the properties of individual stars using data presented in this table.

\section{Convergent point and membership analysis}

In the following we use the $\mathrm{CP}$ search method to identify a reliable moving group in the sample of stars listed in Table 3 that will be the starting point of our traceback analysis (see Sect. 6) to determine the dynamical age of the association.

\subsection{Convergent point}

To accurately determine the $\mathrm{CP}$ position and perform a membership analysis of TWA stars we apply our new CP search method (Galli et al. 2012) to the proper motion data given in Table 3. The method takes the velocity dispersion and mean distance of the moving group as input parameters. The intrinsic velocity dispersion of TWA is expected to be $\sigma_{v} \leq 1 \mathrm{~km} \mathrm{~s}^{-1}$ and the mean distance is $d \simeq 50 \mathrm{pc}$ (see Mamajek 2005). While the velocity dispersion term in the $\mathrm{CP}$ analysis allows us to identify those group members that do not show strict convergence to the $\mathrm{CP}$, it also drives the method to include some additional stars that do not belong to the moving group (de Bruijne 1999a; Galli et al. 2012). Thus, to define a secure group of comoving 
C. Ducourant et al.: The TW Hydrae association: trigonometric parallaxes and kinematic analysis
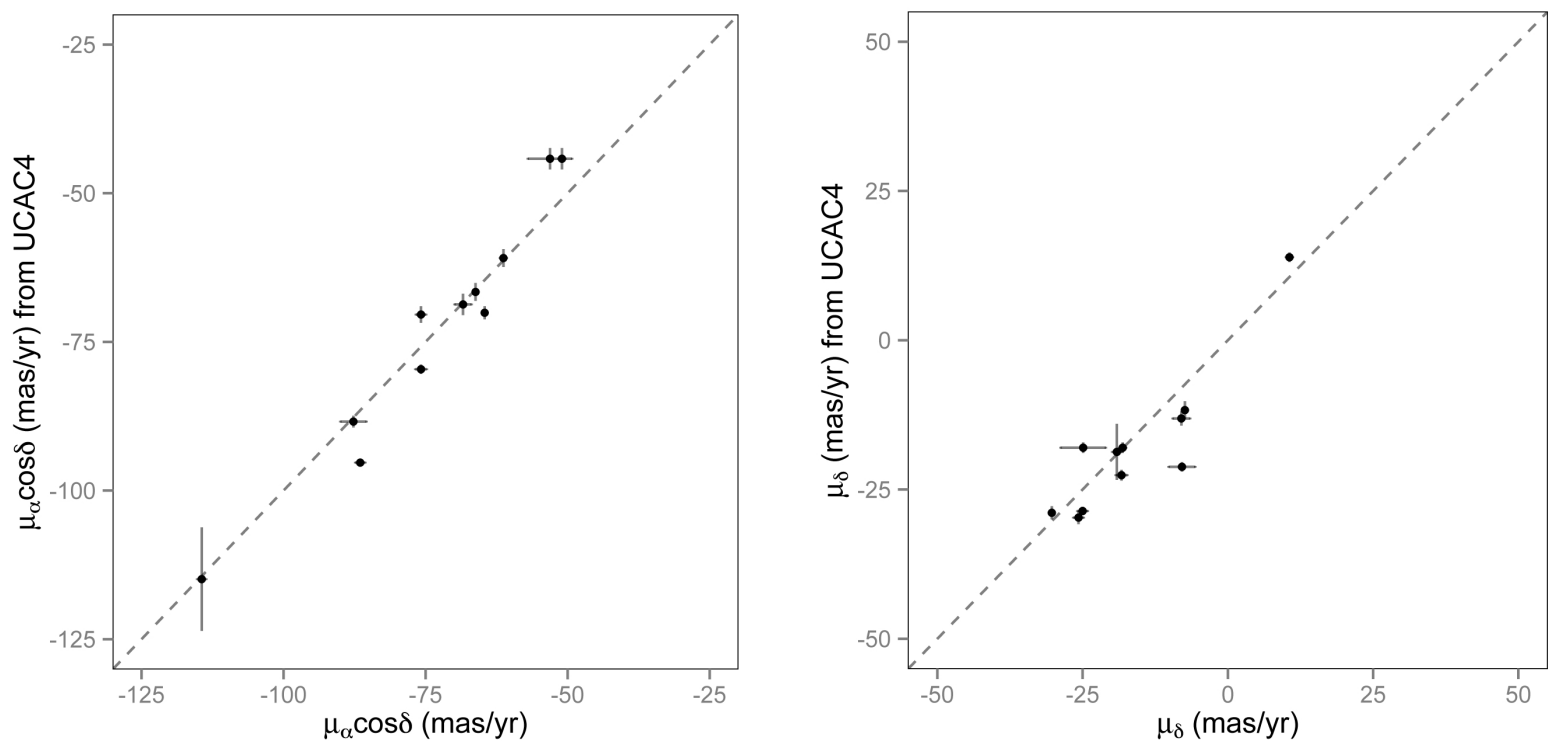

Fig. 2. Comparison of the proper motions derived in our work with those from UCAC4. The dotted line indicates the expected results for perfect correlation. In the left panel, abscissa entries are right ascension values from Col. 6 in Table 1, and in the right panel declination values from Col. 7.

Table 2. Photometry of the TWA stars observed in this work (except TWA 1).

\begin{tabular}{lccccccc}
\hline \hline Star & $\begin{array}{c}V \\
(\mathrm{mag})\end{array}$ & $\begin{array}{c}R \\
(\mathrm{mag})\end{array}$ & $\begin{array}{c}\text { Gunn } i \\
(\mathrm{mag})\end{array}$ & $\begin{array}{c}J \text { (2MASS) } \\
(\mathrm{mag})\end{array}$ & $\begin{array}{c}H(2 \mathrm{MASS}) \\
(\mathrm{mag})\end{array}$ & $\begin{array}{c}K \text { (2MASS) } \\
(\mathrm{mag})\end{array}$ & Multiplicity \\
\hline TWA 2 & $11.10 \pm 0.03$ & $10.04 \pm 0.04$ & $8.91 \pm 0.05$ & 7.629 & 6.927 & 6.710 & $\mathrm{~B}$ \\
TWA 5 & $11.50 \pm 0.03$ & $10.40 \pm 0.04$ & $9.19 \pm 0.05$ & 7.669 & 6.987 & 6.745 & $\mathrm{~T}$ \\
TWA 7 & $11.78 \pm 0.03$ & $10.61 \pm 0.03$ & $9.43 \pm 0.04$ & 7.792 & 7.125 & 6.899 & \\
TWA 8A & $12.33 \pm 0.03$ & $11.18 \pm 0.04$ & $9.88 \pm 0.05$ & 8.337 & 7.663 & 7.430 & $\mathrm{~B}$ \\
TWA 8B & $18.8^{*}$ & $16.6^{*}$ & $15.3^{*}$ & 9.837 & 9.276 & 9.012 & $\mathrm{~B}$ \\
TWA 10 & $12.91 \pm 0.03$ & $11.74 \pm 0.03$ & $10.45 \pm 0.04$ & 9.122 & 8.477 & 8.186 & \\
TWA 12 & $12.94 \pm 0.07$ & $11.81 \pm 0.06$ & $10.58 \pm 0.06$ & 8.999 & 8.334 & 8.053 & \\
TWA 21 & $9.85 \pm 0.07$ & $9.29 \pm 0.06$ & $8.75 \pm 0.06$ & 7.870 & 7.353 & 7.194 & \\
TWA 23 & $12.69 \pm 0.03$ & $11.45 \pm 0.03$ & $10.07 \pm 0.04$ & 8.618 & 8.025 & 7.751 & \\
TWA 26 & $17.25 \pm 0.02$ & $16.84 \pm 0.03$ & $16.46 \pm 0.05$ & 12.686 & 11.996 & 11.503 & \\
\hline
\end{tabular}

Notes. For each star we provide the $V, R, I$ magnitudes derived in this paper, 2MASS $J H K$ photometry (Cutri et al. 2003), and comments on the multiplicity (B for binary and T for triple) of targets as summarized in Appendix A of Messina et al. (2010). The symbol “*” denotes rough photometry.

stars we consider in a first step $\sigma_{v}=0 \mathrm{~km} \mathrm{~s}^{-1}$ and run the CP search method on the sample of classical TWA stars (TWA 1 TWA 34). The results of membership analysis can also be understood in terms of the stop parameter $\epsilon_{\min }$ (see Galli et al. 2012, for more details) that should allow us to find the largest number of moving group members with the least contamination by field stars. To better compare our results with the previous CP analysis of TWA stars performed by Mamajek (2005) we adopt a rejection threshold of $5 \%$ following his procedure. Doing so, we find a moving group of 18 stars (TWA 1, TWA 3, TWA 4, TWA 7, TWA 12, TWA 13A, TWA 13B, TWA 15A, TWA 15B, TWA 21, TWA 23, TWA 26, TWA 29, TWA 30A, TWA 30B, TWA 32, TWA 33, and TWA 34) that shows strict convergence and yields the best $\mathrm{CP}$ estimate to date for TWA located at

$\left(\alpha_{\mathrm{cp}}, \delta_{\mathrm{cp}}\right)=\left(102.4^{\circ},-27.3^{\circ}\right) \pm\left(1.4^{\circ}, 0.6^{\circ}\right)$,

with chi-squared statistics $\chi_{\text {red }}^{2}=1.1$ (i.e., $\chi^{2} / v=17.4 / 16$ ).

We estimate the velocity dispersion of the moving group using Eq. (19) of de Bruijne (1999b) that is given by

$\overline{\mu_{\perp}^{2}}=\overline{\left(A^{-1} \pi \sigma_{v}\right)^{2}}+\overline{\sigma_{\mu_{\perp}}^{2}}$, where $A=4.74047 \mathrm{~km} \mathrm{yr} / \mathrm{s}$ is the ratio of one astronomical unit in $\mathrm{km}$ to the number of seconds in one Julian year. We compute $\mu_{\perp}$, the stellar proper motion component directed perpendicular to the great circle that joins the star and the $\mathrm{CP}$, using the following transformation

$\left(\begin{array}{c}\mu_{\|} \\ \mu_{\perp}\end{array}\right)=\left(\begin{array}{cc}\sin \theta & \cos \theta \\ -\cos \theta & \sin \theta\end{array}\right)\left(\begin{array}{c}\mu_{\alpha} \cos \delta \\ \mu_{\delta}\end{array}\right)$,

where $\theta$ is given by (see also Galli et al. 2012; de Bruijne 1999a)

$\tan \theta=\frac{\sin \left(\alpha_{\mathrm{cp}}-\alpha\right)}{\cos \delta \tan \delta_{\mathrm{cp}}-\sin \delta \cos \left(\alpha_{\mathrm{cp}}-\alpha\right)}$.

To do so, we assume that all stars ${ }^{1}$ in Table 3 with known trigonometric parallax are candidate members of the association. Then we come back to Eq. (1) and set $\sigma_{\mu_{\perp}}=0$ which is consistent with estimating an upper limit for the velocity dispersion of

1 TWA 22 was not considered in this analysis, because previous studies (Mamajek 2005; Barrado Y Navascués 2006; Teixeira et al. 2009) strongly suggest that this star is not a TWA member. 
Table 3. Selected astrometric data for the 34 TWA stars (and stellar systems).

\begin{tabular}{|c|c|c|c|c|c|c|c|c|c|c|c|c|c|}
\hline $\begin{array}{l}\text { (1) } \\
\text { Star }\end{array}$ & $\begin{array}{c}(2) \\
\alpha \\
(\mathrm{h}: \mathrm{m}: \mathrm{s})\end{array}$ & $\begin{array}{c}(3) \\
\delta \\
\left({ }^{\circ}, \prime \prime\right)\end{array}$ & $\begin{array}{c}(4) \\
\sigma_{\alpha} \\
(\mathrm{mas})\end{array}$ & $\begin{array}{c}(5) \\
\sigma_{\delta} \\
(\mathrm{mas})\end{array}$ & $\begin{array}{c}(6) \\
\mu_{\alpha} \cos \delta \\
(\mathrm{mas} / \mathrm{yr})\end{array}$ & $\begin{array}{c}(7) \\
\mu_{\delta} \\
(\mathrm{mas} / \mathrm{yr}) \\
\end{array}$ & $\begin{array}{l}\text { (8) } \\
\text { Ref. }\end{array}$ & $\begin{array}{c}(9) \\
V_{\mathrm{r}} \\
\left(\mathrm{km} \mathrm{s}^{-1}\right) \\
\end{array}$ & $\begin{array}{l}\text { (10) } \\
\text { Ref. }\end{array}$ & $\begin{array}{c}(11) \\
\pi \\
\text { (mas) }\end{array}$ & $\begin{array}{l}\text { (12) } \\
\text { Ref. }\end{array}$ & $\begin{array}{c}(13) \\
\text { CP } \\
\text { Member. }\end{array}$ & $\begin{array}{l}(14) \\
\text { Traceback } \\
\text { Age deter. }\end{array}$ \\
\hline TWA 1 & 110151.905 & -344217.03 & 05 & 04 & $-73.4 \pm 2.3$ & $-17.5 \pm 2.3$ & 1 & $12.66 \pm 0.22$ & 7 & $18.6 \pm 2.1$ & 12 & $\mathrm{Y}$ & + \\
\hline TWA 2 & 110913.798 & -300139.88 & 16 & 13 & $-88.4 \pm 1.0$ & $-21.2 \pm 0.8$ & 2 & $10.58 \pm 0.51$ & 8 & $24.0 \pm 4.8$ & 3 & $\mathrm{Y}$ & + \\
\hline TWA 3 & 111027.894 & -373151.97 & 14 & 15 & $-105.9 \pm 0.9$ & $-17.3 \pm 1.0$ & 2 & $9.52 \pm 0.86$ & 8 & $28.3 \pm 1.6^{*}$ & 3 & $\mathrm{Y}$ & + \\
\hline TWA 4 & 112205.288 & -244639.74 & 04 & 18 & $-91.7 \pm 1.6$ & $-31.1 \pm 1.4$ & 1 & $9.20 \pm 0.10$ & 7 & $22.3 \pm 2.3$ & 12 & $\mathrm{Y}$ & + \\
\hline TWA 5 & 113155.260 & -343627.25 & 10 & 12 & $-79.6 \pm 0.8$ & $-22.6 \pm 0.9$ & 2 & $13.30 \pm 2.00$ & 8 & $20.5 \pm 2.4$ & 3 & Y & $\mathrm{r}$ \\
\hline TWA 6 & 101828.701 & -315002.86 & 14 & 12 & $-55.0 \pm 1.2$ & $-19.8 \pm 1.0$ & 2 & $16.90 \pm 5.00$ & 15 & $14.9 \pm 4.4^{*}$ & 3 & $\mathrm{Y}$ & $\mathrm{r}$ \\
\hline TWA 7 & 104230.100 & -334016.28 & 128 & 63 & $-114.4 \pm 0.8$ & $-19.1 \pm 0.8$ & 3 & $12.21 \pm 0.24$ & 8 & $29.0 \pm 2.1$ & 3 & $\mathrm{Y}$ & + \\
\hline TWA 8A & 113241.263 & -265155.99 & 09 & 09 & $-90.6 \pm 0.9$ & $-32.1 \pm 0.8$ & 4 & $8.34 \pm 0.48$ & 8 & $23.4 \pm 2.0$ & 3 & $\mathrm{Y}$ & + \\
\hline TWA 8B & 113241.172 & -265209.13 & 33 & 78 & $-93.2 \pm 1.3$ & $-27.5 \pm 1.2$ & 4 & $8.93 \pm 0.27$ & 8 & $25.9 \pm 2.0$ & 3 & $\mathrm{Y}$ & + \\
\hline TWA 9A & 114824.229 & -372849.11 & 06 & 03 & $-55.4 \pm 2.3$ & $-17.7 \pm 2.3$ & 1 & $9.46 \pm 0.38$ & 7 & $21.4 \pm 2.5$ & 12 & $\mathrm{Y}$ & $\mathrm{r}$ \\
\hline TWA 9B & 114823.732 & -372848.50 & 27 & 27 & $-51.0 \pm 0.6$ & $-18.1 \pm 0.6$ & 3 & $11.30 \pm 2.00$ & 15 & $19.2 \pm 1.1$ & 3 & $\mathrm{Y}$ & $\mathrm{r}$ \\
\hline TWA 10 & 123504.254 & -413638.64 & 16 & 14 & $-64.6 \pm 0.4$ & $-30.3 \pm 0.4$ & 3 & $6.75 \pm 0.40$ & 8 & $16.2 \pm 1.0$ & 3 & Y? & - \\
\hline TWA 11A & 123601.031 & -395210.23 & 02 & 02 & $-53.3 \pm 1.3$ & $-21.2 \pm 1.1$ & 1 & $9.40 \pm 2.30$ & 7 & $13.7 \pm 0.3$ & 12 & $\mathrm{Y}$ & + \\
\hline TWA 11B & 123601.031 & -395210.23 & 02 & 02 & .............. & ........... & - & $9.00 \pm 1.00$ & 7 & ........... & - & $\mathrm{Y}$ & - \\
\hline TWA 11C & 123548.939 & -395024.50 & 26 & 26 & $-48.6 \pm 1.7$ & $-21.3 \pm 1.6$ & 2 & ........... & - & $14.5 \pm 0.5$ & 5 & $\mathrm{Y}$ & - \\
\hline TWA 12 & 112105.484 & -384516.51 & 16 & 17 & $-66.6 \pm 1.5$ & $-11.7 \pm 1.5$ & 2 & $13.12 \pm 1.59$ & 8 & $15.4 \pm 1.7$ & 3 & $\mathrm{Y}$ & + \\
\hline TWA $13 \mathrm{~A}$ & 112117.219 & -344645.47 & 13 & 16 & $-66.4 \pm 2.4$ & $-12.5 \pm 1.8$ & 5 & $11.67 \pm 0.64$ & 7 & $18.0 \pm 0.7$ & 5 & $\mathrm{Y}$ & + \\
\hline TWA 13B & 112117.446 & -344649.83 & 13 & 16 & $-68.0 \pm 3.1$ & $-11.0 \pm 2.7$ & 5 & $12.57 \pm 0.50$ & 7 & $16.8 \pm 0.7$ & 5 & $\mathrm{Y}$ & + \\
\hline TWA 14 & 111326.221 & -452342.75 & 14 & 14 & $-43.9 \pm 1.4$ & $-7.4 \pm 1.4$ & 2 & $15.83 \pm 2.00$ & 8 & $10.4 \pm 1.2$ & 5 & $\mathrm{Y}$ & + \\
\hline TWA $15 A$ & 123420.649 & -481513.48 & 17 & 17 & $-37.5 \pm 2.4$ & $-10.4 \pm 2.0$ & 5 & $11.20 \pm 2.00$ & 15 & $9.1 \pm 1.7$ & 5 & $\mathrm{Y}$ & + \\
\hline TWA 15B & 123420.473 & -481519.59 & 22 & 17 & $-36.5 \pm 2.9$ & $-9.9 \pm 2.8$ & 5 & $10.03 \pm 1.66$ & 8 & $8.6 \pm 1.6$ & 5 & $\mathrm{Y}$ & + \\
\hline TWA 16 & 123456.303 & -453807.63 & 18 & 11 & $-47.5 \pm 1.3$ & $-20.2 \pm 0.8$ & 2 & $9.01 \pm 0.42$ & 8 & $12.8 \pm 0.5$ & 5 & Y? & - \\
\hline TWA 17 & 132045.388 & -461137.72 & 16 & 10 & $-31.3 \pm 1.1$ & $-17.7 \pm 1.0$ & 2 & $4.60 \pm 6.00$ & 15 & .......... & - & $\mathrm{N}$ & - \\
\hline TWA 18 & 132137.225 & -442151.84 & 14 & 14 & $-32.1 \pm 1.1$ & $-20.4 \pm 1.1$ & 2 & $6.90 \pm 3.00$ & 15 & ........... & - & $\mathrm{N}$ & - \\
\hline TWA 19A & 114724.545 & -495303.01 & 03 & 03 & $-33.7 \pm 1.1$ & $-9.1 \pm 1.1$ & 1 & $11.50 \pm 3.80$ & 7 & $10.9 \pm 1.3$ & 12 & $\mathrm{~N}$ & - \\
\hline TWA 19B & 114720.642 & -495304.31 & 44 & 47 & $-21.6 \pm 1.7$ & $-23.4 \pm 1.6$ & 4 & $15.20 \pm 2.00$ & 15 & ............ & - & $\mathrm{N}$ & - \\
\hline TWA 20 & 123138.068 & -455859.47 & 12 & 12 & $-63.5 \pm 1.1$ & $-27.8 \pm 1.1$ & 2 & $8.10 \pm 4.00$ & 15 & $12.9 \pm 0.6$ & 5 & Y? & - \\
\hline TWA 21 & 101314.774 & -523053.95 & 16 & 10 & $-60.9 \pm 1.5$ & $13.9 \pm 0.8$ & 2 & $17.50 \pm 0.80$ & 10 & $19.8 \pm 1.4$ & 3 & $\mathrm{Y}$ & $\mathrm{r}$ \\
\hline TWA 22 & 101726.905 & -535426.42 & 18 & 18 & $-175.8 \pm 0.8$ & $-21.3 \pm 0.8$ & 13 & $13.57 \pm 0.26$ & 8 & $57.0 \pm 0.7$ & 13 & $\mathrm{~N}$ & - \\
\hline TWA 23 & 120727.377 & -324700.25 & 18 & 15 & $-75.8 \pm 0.9$ & $-25.7 \pm 0.9$ & 3 & $8.52 \pm 1.20$ & 8 & $20.6 \pm 1.8$ & 3 & $\mathrm{Y}$ & + \\
\hline TWA 24 & 120941.861 & -585445.08 & 12 & 18 & $-35.2 \pm 1.8$ & $-14.9 \pm 2.1$ & 2 & $11.90 \pm 0.90$ & 10 & .............. & - & $\mathrm{N}$ & - \\
\hline TWA 25 & 121530.723 & -394842.59 & 12 & 13 & $-73.2 \pm 0.8$ & $-27.7 \pm 0.8$ & 2 & $9.20 \pm 2.10$ & 10 & $18.5 \pm 1.2$ & 5 & Y? & - \\
\hline TWA 26 & 113951.140 & -315921.50 & 60 & 60 & $-93.3 \pm 0.5$ & $-27.5 \pm 0.5$ & 3 & $11.60 \pm 2.00$ & 14 & $26.2 \pm 1.1$ & 3 & $\mathrm{Y}$ & $\mathrm{r}$ \\
\hline TWA 27 & 120733.467 & -393254.00 & 60 & 60 & $-64.2 \pm 0.4$ & $-22.6 \pm 0.4$ & 3 & $11.02 \pm 2.00$ & 14 & $19.1 \pm 0.4$ & 3 & $\mathrm{Y}$ & $\mathrm{r}$ \\
\hline TWA 28 & 110209.833 & -343035.53 & 60 & 60 & $-67.2 \pm 0.6$ & $-14.0 \pm 0.6$ & 13 & ............ & - & $18.1 \pm 0.5$ & 13 & $\mathrm{Y}$ & - \\
\hline TWA 29 & 124513.798 & -442847.63 & 44 & 38 & $-71.0 \pm 7.0$ & $-23.0 \pm 3.0$ & 3 & ........... & - & $12.7 \pm 2.1$ & 5 & $\mathrm{Y}$ & - \\
\hline TWA 30A & 113218.315 & -301951.85 & 20 & 20 & $-87.8 \pm 1.3$ & $-25.2 \pm 1.3$ & 2 & $12.30 \pm 1.50$ & 11 & $18.0 \pm 2.2^{*}$ & 3 & $\mathrm{Y}$ & $\mathrm{r}$ \\
\hline TWA 30B & 113216.921 & -301810.53 & 21 & 21 & $-83.0 \pm 9.0$ & $-30.0 \pm 9.0$ & 11 & $12.00 \pm 3.00$ & 11 & $17.8 \pm 4.8^{*}$ & 3 & $\mathrm{Y}$ & $\mathrm{r}$ \\
\hline TWA 31 & 120716.547 & -323022.36 & 10 & 10 & $-42.0 \pm 6.0$ & $-36.0 \pm 3.0$ & 6 & $10.47 \pm 0.41$ & 8 & ……..... & - & $\mathrm{N}$ & - \\
\hline TWA 32 & 122651.367 & -331612.54 & 32 & 79 & $-59.7 \pm 2.4$ & $-22.9 \pm 2.2$ & 4 & $7.15 \pm 0.26$ & 8 & $13.0 \pm 0.7 *$ & 3 & $\mathrm{Y}$ & + \\
\hline TWA 33 & 113933.846 & -304000.34 & 50 & 45 & $-73.3 \pm 2.9$ & $-25.4 \pm 2.6$ & 4 & ……..... & - & $19.0 \pm 1.2^{*}$ & 3 & $\mathrm{Y}$ & - \\
\hline TWA 34 & 102845.790 & -283037.53 & 38 & 35 & $-68.6 \pm 2.7$ & $-11.4 \pm 2.5$ & 4 & $\ldots \ldots \ldots \ldots$ & - & $20.0 \pm 1.3^{*}$ & 3 & $\mathrm{Y}$ & - \\
\hline
\end{tabular}

Notes. In Cols. 2-5 we provide for each star its position $($ epoch $=2000.0)$ from UCAC4 whenever available otherwise from SPM4 or 2MASS. The proper motion, radial velocity, parallax, and the corresponding references are given in Cols. 6-12. Parallaxes with a "*” symbol are kinematic parallaxes derived in Sect. 4.3. Column 13 gives the membership status derived from the CP search method $(\mathrm{Y}=$ member, $\mathrm{N}=$ non-member, $\mathrm{Y} ?=$ possible member) as defined in Sect. 4.4. Column 14 summarizes the participation of CP members to the traceback age determination (see Sect. 5.2 for details) (+ = participation, $r=$ rejected, - : not member or lack of data).

References. (1) Tycho-2 (Høg et al. 2000); (2) UCAC4 (Zacharias et al. 2013); (3) This paper or Ducourant et al. (2008); (4) SPM4 (Girard et al. 2011); (5) Weinberger et al. (2013); (6) Schneider et al. (2012a); (7) Torres et al. (2003); (8) Shkolnik et al. (2011); (9) Torres et al. (2008); (10) Song et al. (2003); (11) Looper et al. (2010a,b); (12) Hipparcos (van Leeuwen 2007); (13) Teixeira et al. (2008, 2009); (14) Mohanty et al. (2003); (15) Reid (2003).

the association for those stars with known trigonometric parallax. The upper limit will allow us to recover as many members as possible in our upcoming $\mathrm{CP}$ analysis with a non-zero velocity dispersion value. A more refined velocity dispersion estimate will be discussed in Sect. 4.4 with our final sample of moving group members. The velocity dispersion $\sigma_{v}$ in Eq. (1) that arises only from the perpendicular motion of stars is estimated iteratively, i.e., for each computed value of $\sigma_{v}$ we recalculate the $\mathrm{CP}$ position. In the first iteration we use $\sigma_{v}=0 \mathrm{~km} \mathrm{~s}^{-1}$ and the $\mathrm{CP}$ solution mentioned above. After a few iterations we converge toward $\sigma_{v} \simeq 0.8 \pm 0.1 \mathrm{~km} \mathrm{~s}^{-1}$.

When we run the CP search method on the initial sample of TWA stars with $\sigma_{v}=0.8 \mathrm{~km} \mathrm{~s}^{-1}$ we end up with a moving group of 30 stars that can be regarded as kinematic members of the association. The associated CP is located at

$\left(\alpha_{\mathrm{cp}}, \delta_{\mathrm{cp}}\right)=\left(100.1^{\circ},-27.1^{\circ}\right) \pm\left(3.0^{\circ}, 1.3^{\circ}\right)$, with chi-squared statistics $\chi_{\text {red }}^{2}=1.2$ (i.e., $\chi^{2} / v=34.6 / 28$ ). We note that the addition of 12 stars (TWA 2, TWA 5, TWA 6, TWA 8A, TWA 8B, TWA 9A, TWA 9B, TWA 11A, TWA 11C, TWA 14, TWA 27, and TWA 28) to the 18 listed in the preceding paragraph shifts the CP position, but both results are still compatible within $1 \sigma$. Our solution is consistent with the $\mathrm{CP}$ derived by Mamajek $(2005),\left(\alpha_{\mathrm{cp}}, \delta_{\mathrm{cp}}\right)=\left(100.5^{\circ},-27.9^{\circ}\right) \pm\left(5.0^{\circ}, 2.3^{\circ}\right)$, who is using a different proper motion data set and CP method. However, our convergent point estimate is more precise which comes naturally from the proper motion data available at the moment.

\subsection{Validation}

In the following we investigate via Monte Carlo simulations the validity of our $\mathrm{CP}$ solution derived with 30 moving group 


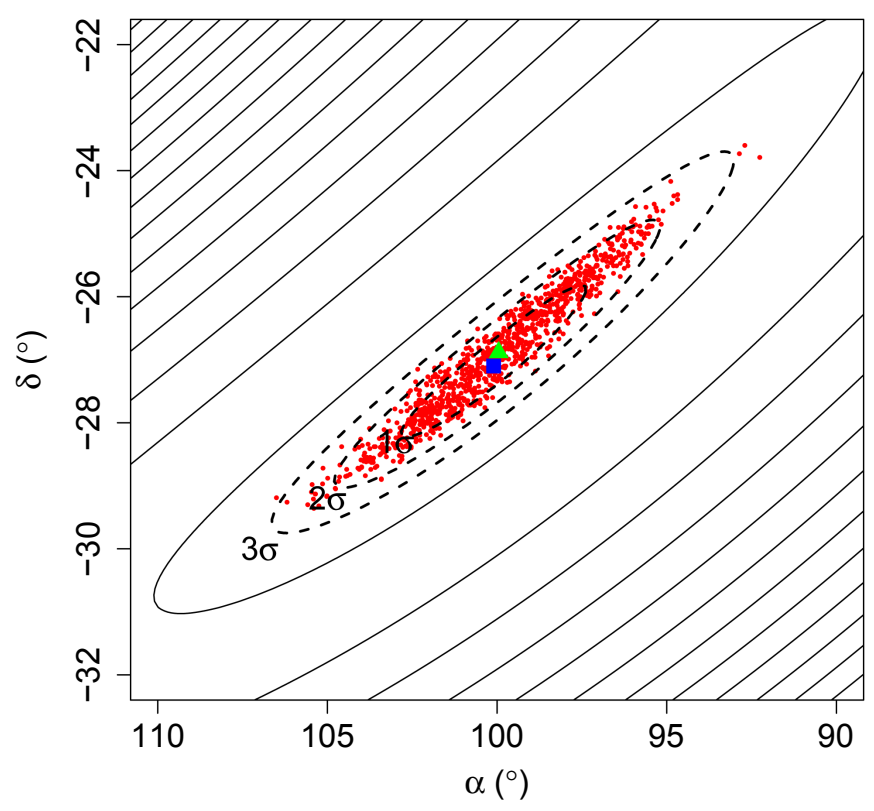

Fig. 3. Location of the simulated CPs for 1000 Monte Carlo realizations (red dots). The solid lines indicate the $X^{2}$ contours and the dashed lines denote the $1 \sigma, 2 \sigma$ and $3 \sigma$ confidence levels for the CP solution with 30 members presented in Sect. 4.1 (blue square). The green triangle indicates the centroid of simulated CPs.

members (see Sect. 4.1). We construct 1000 synthetic samples of TWA by resampling the stellar proper motions from a Gaussian distribution where the mean and variance correspond to the proper motion and its uncertainty given in Table 3. For each simulated data set we run the CP search method (with $d=50 \mathrm{pc}$ and $\sigma_{v}=0.8 \mathrm{~km} \mathrm{~s}^{-1}$ ) and compute the CP location. The CP derived in Sect. 4.1 is perfectly consistent with the centroid of simulated CPs (see Fig. 3) that is located at

$\left(\alpha_{\mathrm{cp}}, \delta_{\mathrm{cp}}\right)=\left(100.0^{\circ},-26.9^{\circ}\right) \pm\left(2.3^{\circ}, 1.0^{\circ}\right)$.

Thus, we conclude that our CP solution presented in Sect. 4.1 is representative of the TWA moving group.

\subsection{Kinematic parallaxes}

When a star belongs to a moving group its proper motion and radial velocity can be used to determine its distance (see, e.g., Mamajek 2005; Bertout \& Genova 2006; Galli et al. 2012). We use this approach to estimate the distance for seven TWA members whose trigonometric parallax is not known in the literature. The kinematic parallax for each group member is given by

$\pi_{\mathrm{kin}}=\frac{A \mu_{\|}}{V_{\mathrm{r}} \tan \lambda}$,

where $\lambda$ is the angular distance from the CP to the star, $V_{\mathrm{r}}$ is the radial velocity, and $\mu_{\|}$is the stellar proper motion component directed toward the CP (as defined in Sect. 4.1).

Among the 30 TWA members identified in our CP analysis only TWA 3, TWA 6, TWA 30A, TWA 30B, TWA 32, TWA 33, and TWA 34 do not have any trigonometric parallax measurement in the literature (and in this paper). We use the procedure described in the preceding paragraph and in Eq. (4) to derive their kinematic parallax (Eq. (5) was used instead when radial velocity measurement was not available; see next paragraph); in Table 3 these values are marked with "**" to distinguish them from other stars with trigonometric parallaxes. This is the first determination of kinematic distance for TWA 30A, TWA 30B, TWA 32, TWA 33, and TWA 34, because they were not included in the previous CP analysis performed by Mamajek (2005).

For the specific case of TWA 3 we found five different radial velocity measurements (see de la Reza et al. 1989; Reid 2003; Mamajek 2005; Torres et al. 2006; Shkolnik et al. 2011). This source is known to be a multiple system (see, e.g., Schneider et al. 2012a); this makes the various radial velocity values found in the literature credible. Since TWA 3 has not been resolved in current astrometric catalogs, the proper motion provided in Table 3 should be regarded as representative of the stellar system. On the other hand, TWA 33 and TWA 34 have been identified as TWA moving group members based on their proper motions and our CP analysis (see Sect. 4.1), but they do not have any measured radial velocity in the literature. In these cases, Eq. (4) cannot be used to compute the stellar kinematic parallax. Thus, we use an alternative approach by assuming that those stars that belong to a moving group share the same space motion. In this case, the approximate parallax of the star is given by

$\pi_{\text {app }}=\frac{A \mu_{\|}}{V_{\text {space }} \sin \lambda}$,

where the group spatial velocity is $V_{\text {space }}=21.5 \pm 1.2 \mathrm{~km} \mathrm{~s}^{-1}$ (to be discussed in Sect. 5). We use this approach to derive an approximate parallax for TWA 3, TWA, 33 and TWA 34. We note that our distance estimates for TWA $33\left(d=53_{-3}^{+3} \mathrm{pc}\right)$ and TWA $34\left(d=50_{-3}^{+3} \mathrm{pc}\right)$ agree with the results presented by Schneider et al. (2012b), but they are more precise as a result of the more precise proper motion data used in the present paper.

\subsection{Membership analysis}

The final membership status of each star considered in this paper is given in Table 3 together with their astrometry. The 30 stars selected by the CP method in our analysis (see Sect. 4.1) are considered TWA moving group members and their membership status is indicated with "Y". Our analysis confirms TWA 11A and TWA $11 \mathrm{C}$ as moving group members while the membership status of the TWA 11B component, whose proper motion is not known, cannot be investigated with the $\mathrm{CP}$ method. However, it seems unlikely that TWA $11 \mathrm{~B}$ is not co-moving with the other components of this multiple system, so we consider TWA 11B to be a group member and mark its membership status with "Y"; we end up with a list of 31 kinematic members.

Among the rejected stars in our $\mathrm{CP}$ analysis we note that TWA 17, TWA 18, TWA 19A, TWA 19B, and TWA 24 have also been excluded as TWA members by Mamajek (2005) because their distances inferred from the CP strategy are more consistent with the Lower Centaurus-Crux (LCC) subgroup of the Scorpius-Centaurus (Sco-Cen) association. That these stars are also rejected in our new and revised $\mathrm{CP}$ solution confirms the previous results. TWA 31 is also rejected by the $\mathrm{CP}$ search method, possibly due to its poor proper motion compared to other TWA members. Its membership status should be rediscussed when more precise measurements become available. Our analysis also confirms that TWA 22 is not a group member as already suggested in previous studies (Mamajek 2005; Barrado Y Navascués 2006; Teixeira et al. 2009). All stars mentioned in this paragraph are therefore considered non-members of the association following our analysis, and their membership status in Table 3 is indicated with " $N$ ". 
On the other hand, TWA 10, TWA 16, TWA 20, and TWA 25 were considered TWA members in Mamajek's analysis, but are rejected by the $\mathrm{CP}$ search method in the present paper. By comparing the stellar proper motions used in both papers we conclude that those used here are more precise than the ones that were available when Mamajek (2005) preformed his CP analysis. When we replace the proper motions given in Table 3 of the present paper for TWA 10, TWA 16, TWA 20, and TWA 25 with the ones listed in Table 1 of Mamajek (2005), and run the CP search method, we observe that these stars can be tolerated in our solution with negligible impact to the CP position. It seems that the large proper motion errors used in Mamajek's $\mathrm{CP}$ analysis have cast doubt on the derived $\mu_{\perp}$ allowing these stars to be considered TWA moving group members. Thus, given the more recent and precise proper motion measurements available now, such as the ones listed in Table 3, we cannot confirm TWA 10, TWA 16, TWA 20, and TWA 25 as TWA members based only on the CP search method. Galli et al. (2012) performed extensive simulations that convincingly demonstrate that more than $80 \%$ of all cluster members can be retrieved using the $\mathrm{CP}$ search method employed in this paper. Although the fraction of cluster members identified with this technique is high, we may have missed a few group members. Additional information for TWA 10, TWA 16, TWA 20, and TWA 25, such as, parallax and radial velocity (see Table 3), spatial location (see Sect. 5), and isochronal age (see Sect. 6) suggest that they are consistent with membership in TWA. Thus, we retain these stars as possible TWA members, and mark their membership status in Table 3, Col. (13) with "Y?" to distinguish them from those group members that were directly identified by the CP search method.

Once we have defined our final list of association members we are now in a better position to determine an accurate value for the velocity dispersion of the group. We estimate the intrinsic velocity dispersion of the association by the distribution of perpendicular velocities $V_{\perp}$ and their errors that arise from the $\mu_{\perp}$ proper motion components. Then, we search for the velocity dispersion $\sigma_{v}$ needed to force our result to $\chi_{\text {red }}^{2}=1$. We estimate the uncertainty of our result by defining the lower and upper limits for the velocity dispersion within an acceptable range of $\chi^{2}$ for a good fit (see, e.g., Gould 2003). We find $\sigma_{v}=0.6_{-0.1}^{+0.2} \mathrm{~km} \mathrm{~s}^{-1}$ using all stars with known trigonometric parallax and marked with "Y" (and not "Y?") in Table 3. Recalculating the CP with $\sigma_{v}=0.6 \mathrm{~km} \mathrm{~s}^{-1}$ has negligible effect on our solution. However, in Sect. 4.3 we derived kinematic parallaxes for another seven members of the association. Now that we have a distance estimate for all 30 moving group members identified in our CP analysis, there is no reason to limit the velocity dispersion analysis to only those 23 stars with known trigonometric parallax. Calculating the velocity dispersion with all 30 moving group members yields instead $\sigma_{v}=0.8_{-0.1}^{+0.2} \mathrm{~km} \mathrm{~s}^{-1}$. We observe that our final velocity dispersion estimate confirms the results discussed in Sect. 4.1 and is identical to the value of $\sigma_{v}=0.8_{-0.2}^{+0.3} \mathrm{~km} \mathrm{~s}^{-1}$ derived by Mamajek (2005) in his analysis ${ }^{2}$.

\section{Space motion and dynamical age}

Makarov et al. (2005) and de la Reza et al. (2006) pioneered the idea of accurately deriving the dynamical age of the TWA. Makarov et al. (2005) investigated the path of several proba-

\footnotetext{
2 It is important to note that the upper limit of $0.8 \mathrm{~km} \mathrm{~s}^{-1}$ given in Sect. 4.1 refers only to the sample of stars in Table 3 with known trigonometric parallax.
}

ble members of the association and showed that five objects form a rapidly expanding association with an expansion age of 4.7 \pm 0.6 My. De la Reza et al. (2006) showed that four HipParcos stars belonging to the association were converging back in time toward a minimum volume corresponding to the dynamical age of the association. This minimum volume was reached at an age of 8.3 Myr. Their different conclusions relied heavily on a small number of objects and on the accuracy of the data they used, in particular radial velocities. Since then, efforts have been dedicated to the measurement of accurate parallaxes and proper motions of TWA stars (Ducourant et al. 2008; Teixeira et al. 2008, 2009; Weinberger et al. 2013) to secure the determination of the dynamical age of the association.

Suspicion of a spread in the ages of the members was raised by several authors. Lawson \& Crause (2005) found evidence for two populations spatially and rotationally distinct, the TWA 1-13 group being younger ( $10 \mathrm{Myr})$ and closer to the Sun while TWA 14-19 would constitute an older ( $\sim 17 \mathrm{Myr})$ and more distant pre-main-sequence population associated instead with the LCC subgroup. Barrado Y Navascués (2006) revisited the age estimation of the association using various methods and notes that the TWA stars might not be coeval and that each age estimation method has its own limitations.

Recently, Weinberger et al. (2013) published 14 parallaxes that greatly increased the number of available distances to TWA stars. They tried to derive a dynamical age for the association by using the astrometric data available, but could not find any convergence back in time and concluded that TWA members have parallel motions. With a new set of available parallaxes and proper motions (from Table 3), we are now in a situation to revisit the spatial motion of TWA stars and the dynamical age of the association.

\subsection{Space motion}

Among the 42 TWA stars (including resolved components) given in Table 3, 31 stars have both known parallaxes (trigonometric or kinematic) and radial velocities. We computed the Galactic positions and velocities of these stars using the procedure described by Johnson \& Soderblom (1987). These results are presented in Table 4 . The Galactic positions $(X, Y, Z)$ are given in a right-handed coordinate system with origin at the Sun where $X$ points toward the Galactic center, $Y$ points in the direction of Galactic rotation, and $Z$ points to the Galactic north pole. The stellar velocity projected on this $X Y Z$ grid defines the $(U, V, W)$ components of the Galactic space velocity.

Obviously, some objects exhibit motions or positions different from the rest. Are these differences a consequence of the history of these stars or of unreliable data? A source of difficulty in this kind of kinematic analysis is the quality and reliability of data, especially of radial velocities. Since many TWA stars are in double or multiple systems, the radial velocity of an individual star, depending on the separation of the components and the period of the system may change noticeably with time (Makarov 2007). This point is of great importance for the following section where we intend to trace stars back in time to find a common origin.

\subsection{Traceback age of TWA}

To determine the traceback age of the association, we considered the 25 stars, kinematic members as determined in Sect. 4.1 (status = "Y" in Table 3 ) that have radial velocity and parallax 
Table 4. Galactic position and space velocity with respect to the Sun for the 31 stars from Table 3 with available parallaxes (trigonometric or kinematic) and radial velocities.

\begin{tabular}{lcccrrr}
\hline \hline Star & $\begin{array}{c}X \\
(\mathrm{pc})\end{array}$ & $\begin{array}{c}Y \\
(\mathrm{pc})\end{array}$ & $\begin{array}{c}Z \\
(\mathrm{pc})\end{array}$ & $\begin{array}{r}U \\
\left(\mathrm{~km} \mathrm{~s}^{-1}\right)\end{array}$ & $\begin{array}{r}V \\
\left(\mathrm{~km} \mathrm{~s}^{-1}\right)\end{array}$ & $\begin{array}{r}W \\
\left(\mathrm{~km} \mathrm{~s}^{-1}\right)\end{array}$ \\
\hline TWA1 & 7.5 & -48.9 & 21.0 & $-12.1 \pm 1.7$ & $-18.5 \pm 0.8$ & $-6.4 \pm 1.4$ \\
TWA2 & 5.1 & -36.5 & 19.5 & $-11.8 \pm 2.6$ & $-16.4 \pm 1.5$ & $-5.1 \pm 2.0$ \\
TWA3 & 6.5 & -31.6 & 12.5 & $-11.9 \pm 0.5$ & $-15.1 \pm 0.8$ & $-5.6 \pm 0.4$ \\
TWA4 & 5.4 & -36.9 & 24.9 & $-12.7 \pm 1.5$ & $-17.5 \pm 1.0$ & $-6.5 \pm 1.2$ \\
TWA5 & 11.3 & -42.6 & 21.0 & $-10.8 \pm 1.7$ & $-20.2 \pm 2.0$ & $-4.3 \pm 1.5$ \\
TWA6 & -1.4 & -62.8 & 23.7 & $-10.9 \pm 3.1$ & $-21.0 \pm 4.9$ & $-8.4 \pm 4.6$ \\
TWA7 & 2.4 & -31.9 & 12.9 & $-13.2 \pm 1.0$ & $-17.0 \pm 0.5$ & $-6.8 \pm 0.8$ \\
TWA8A & 7.5 & -35.1 & 23.1 & $-11.6 \pm 1.1$ & $-16.6 \pm 0.9$ & $-6.1 \pm 1.0$ \\
TWA8B & 6.7 & -31.8 & 20.9 & $-11.1 \pm 1.0$ & $-16.0 \pm 0.7$ & $-4.2 \pm 0.7$ \\
TWA9A & 14.2 & -40.4 & 18.8 & $-6.4 \pm 1.2$ & $-14.4 \pm 0.8$ & $-2.6 \pm 0.9$ \\
TWA9B & 15.8 & -45.0 & 21.0 & $-5.9 \pm 0.8$ & $-16.3 \pm 1.8$ & $-2.4 \pm 0.9$ \\
TWA10 & 28.5 & -50.0 & 22.3 & $-10.9 \pm 0.9$ & $-17.7 \pm 0.8$ & $-7.0 \pm 0.6$ \\
TWA11A & 33.3 & -58.4 & 28.4 & $-9.6 \pm 1.2$ & $-19.3 \pm 1.9$ & $-4.2 \pm 1.0$ \\
TWA12 & 14.9 & -58.8 & 23.1 & $-13.3 \pm 1.9$ & $-20.0 \pm 1.7$ & $-5.5 \pm 1.3$ \\
TWA13A & 11.1 & -49.3 & 23.1 & $-11.4 \pm 0.8$ & $-17.5 \pm 0.7$ & $-3.9 \pm 0.6$ \\
TWA13B & 11.9 & -52.8 & 24.7 & $-12.8 \pm 1.1$ & $-18.9 \pm 0.7$ & $-3.9 \pm 0.8$ \\
TWA14 & 24.9 & -89.9 & 23.5 & $-11.8 \pm 2.0$ & $-21.9 \pm 2.1$ & $-6.5 \pm 1.4$ \\
TWA15A & 53.2 & -92.1 & 27.6 & $-10.3 \pm 3.3$ & $-20.4 \pm 2.7$ & $-3.7 \pm 1.7$ \\
TWA15B & 56.3 & -97.5 & 29.2 & $-11.4 \pm 3.4$ & $-19.7 \pm 2.7$ & $-4.1 \pm 2.0$ \\
TWA16 & 37.2 & -64.7 & 23.0 & $-9.2 \pm 0.7$ & $-18.2 \pm 0.6$ & $-5.6 \pm 0.4$ \\
TWA19A & 34.4 & -83.0 & 18.6 & $-7.3 \pm 2.0$ & $-16.9 \pm 3.5$ & $-5.0 \pm 1.3$ \\
TWA20 & 36.4 & -64.7 & 22.3 & $-14.1 \pm 2.1$ & $-20.8 \pm 3.4$ & $-9.2 \pm 1.3$ \\
TWA21 & 8.8 & -49.6 & 2.8 & $-10.6 \pm 1.0$ & $-19.9 \pm 0.8$ & $-4.6 \pm 0.5$ \\
TWA22 & 3.5 & -17.2 & 0.7 & $-8.2 \pm 0.2$ & $-15.9 \pm 0.3$ & $-9.0 \pm 0.1$ \\
TWA23 & 16.1 & -39.2 & 23.7 & $-10.2 \pm 1.2$ & $-17.1 \pm 1.3$ & $-3.9 \pm 0.9$ \\
TWA25 & 21.5 & -45.1 & 20.7 & $-10.5 \pm 1.3$ & $-18.6 \pm 1.9$ & $-5.6 \pm 1.0$ \\
TWA26 & 9.1 & -32.3 & 18.2 & $-9.9 \pm 0.7$ & $-18.3 \pm 1.7$ & $-3.2 \pm 1.0$ \\
TWA27 & 19.5 & -44.2 & 20.1 & $-8.0 \pm 0.8$ & $-18.2 \pm 1.7$ & $-3.6 \pm 0.8$ \\
TWA30A & 10.2 & -43.2 & 25.1 & $-13.4 \pm 2.2$ & $-20.8 \pm 1.9$ & $-5.3 \pm 1.8$ \\
TWA30B & 10.3 & -43.6 & 25.4 & $-12.1 \pm 4.6$ & $-20.6 \pm 4.0$ & $-6.2 \pm 4.1$ \\
TWA32 & 27.0 & -52.8 & 33.3 & $-11.4 \pm 1.6$ & $-18.0 \pm 1.4$ & $-4.7 \pm 1.1$ \\
\hline & & & & & &
\end{tabular}

measurements (TWA 1, TWA 2, TWA 3, TWA 4, TWA 5, TWA 6, TWA 7, TWA 8A, TWA 8B, TWA 9A, TWA 9B, TWA 11A, TWA 12, TWA 13A, TWA 13B, TWA 14, TWA 15A, TWA 15B, TWA 21, TWA 23, TWA 26, TWA 27, TWA 30A, TWA 30B, and TWA 32).

We then considered the present day positions of these objects and computed their location backward in time with a step of $0.1 \mathrm{Myr}$ for a period of $20 \mathrm{Myr}$. To characterize the extent of the association at each epoch we computed the mean coordinates $(\bar{X}, \bar{Y}, \bar{Z})$ of the group and their associated standard deviation about the mean $\left(\sigma_{X}, \sigma_{Y}, \sigma_{Z}\right)$. We defined the typical radius of the association as $\mathrm{rad}=\frac{1}{3}\left(\sigma_{X}+\sigma_{Y}+\sigma_{Z}\right)$ and searched for the epoch minimizing this quantity. This radius should be representative of the global spread of stars around the mean.

The examination of the evolution with time of the distance of each object from the mean of the group revealed that several objects systematically drifted away from the center of the association back in time. This is the case for TWA 5, TWA 6 , TWA 9A, TWA 9B, TWA 21, TWA 26, TWA 27, TWA 30A, and TWA 30B. Several reasons may explain this behavior, such as corrupted data (the most likely), contamination by nonmembers, or noncoevality with others stars.

Eliminating these 9 discrepant stars leaves us with a sample of 16 stars (TWA 1, TWA 2, TWA 3, TWA 4, TWA 7, TWA 8A, TWA 8B, TWA 11A, TWA 12, TWA 13A, TWA 13B, TWA 14, TWA 15A, TWA 15B, TWA 23, and TWA 32). We will designate these converging members traceback core stars. Their participation in the determination of the traceback age is indicated in Table 3, Col. (14) : “+” for traceback core stars, "r" for objects rejected because they were systematically drifting away from TWA center, and "-" for non members as defined by CP analysis or when data were missing for space velocity calculation.

Assuming that our sample of stars may be contaminated by nonmembers and to get rid of the particular influence of each star we applied a Jackknife resampling technique to our list of 16 core-stars eliminating randomly three objects $(20 \%$ of the sample). We generated 2000 random lists of 13 stars taken from our 16 corestars and calculated for each the epoch of convergence when rad is minimum. Each possible configuration of 13 stars drawn from 16 was represented about three or four times in our trials. The mean of the epochs obtained and the dispersion about the mean correspond to the traceback age of TWA $t \simeq-7.5 \pm 0.7$ Myr. In Fig. 4 we present the evolution of the radius of the association rad as a function of time for the 2000 configurations and in Fig. 5 the repartition of the epochs corresponding to the minimum radius.

One notices several groups of curves in Fig. 4. The upper one contains the most configurations. The lowest ones correspond to samples where TWA 15A or/and TWA 15B are absent. These stars have the smallest parallaxes of any in Table 3, so that their non-inclusion naturally diminishes the radius of the association. One could question whether these stars are members of TWA and instead place them in LCC which is located behind the TWA. However, TWA 15A and 15B are consistent with TWA membership according to our $\mathrm{CP}$ analysis and their evolutionary ages derived in Sect. 6 are more consistent with the mean 


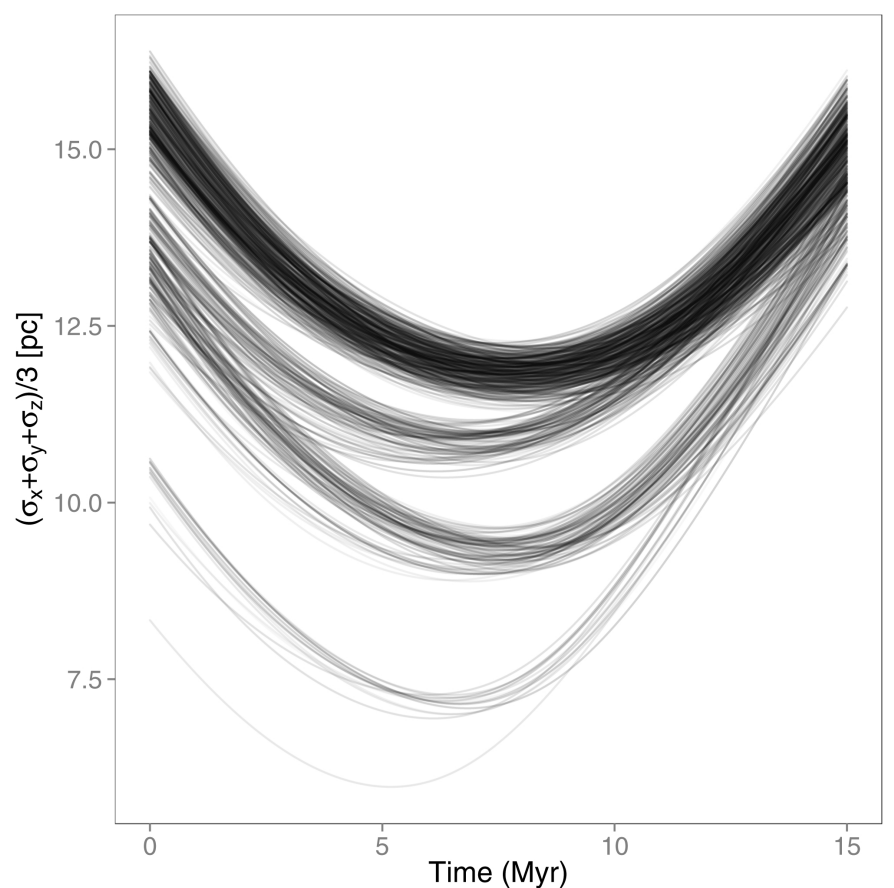

Fig. 4. Evolution back in time of the radius rad of the 2000 resampled lists of 13 TWA corestars.

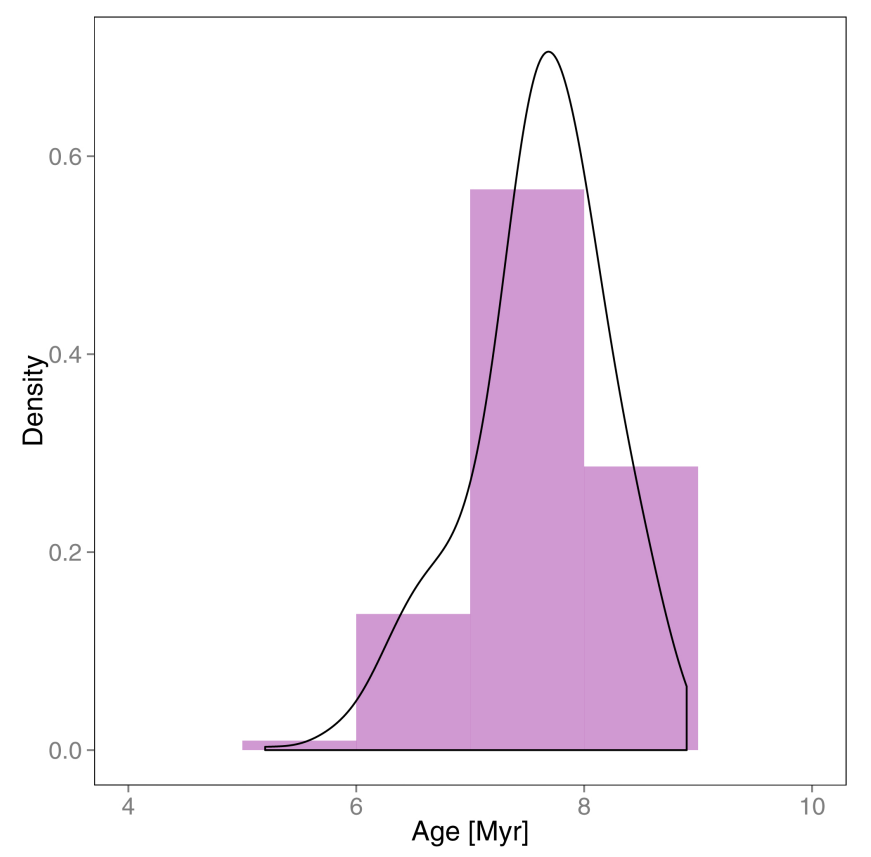

Fig. 5. Distribution of epochs corresponding to the minimum radius of the 2000 resampled lists of 13 TWA corestars as indicated by a histogram (in purple) and a kernel density estimator (in black). The mean of the distribution and dispersion about the mean indicate a traceback age for the association of $t \simeq-7.5 \pm 0.7 \mathrm{Myr}$.

evolutionary age of TWA than with that of LCC (17 Myr, Chen et al. 2011); even if LCC is not as old as $17 \mathrm{Myr}$, it is older than TWA (Fig. 2 in Song et al. 2012). Moreover, a chi-square test applied to the UVW velocities of these two objects to test their adequation to the mean velocities of TWA or LCC systematically better agrees with a kinematic membership to TWA. Therefore, we retain TWA15A and B in our list of core traceback stars. A traceback strategy applied to the list excluding TWA 15A and TWA $15 B$ leads to a traceback age of $-6.3+/-0.8$ Myr.

The weighted mean UVW velocities of the 16 traceback core stars are $(-11.7,-17.3,-5.0) \pm(0.9,1.3,1.0) \mathrm{km} \mathrm{s}^{-1}$. These values are in good agreement with various published values (e.g., Torres et al. 2008; Weinberger et al. 2013) and confirms that our sample of traceback core stars consists of genuine TWA members. These converging members lie at a mean distance $d \simeq 61 \mathrm{pc}$ (ranging from $34.6 \mathrm{pc}$ to $116.3 \mathrm{pc}$ ).

The age derived in this work can be compared to similar investigations via backtracking. Makarov et al. (2005) derived an expansion age of 4.7 Myr using Hipparcos data for TWA 1, 4, 11 and two other young nearby stars (HD 139084 and HD 220476). De la Reza et al. (2006) derived an expansion age of $8.3 \pm 0.8 \mathrm{Myr}$ using Hipparcos data for TWA 1, 4, 11 , and 19. These two determinations, based essentially on the same astrometric data (HIPPARCos), differ by the selection of stars (inclusion or not of TWA 19) and inclusion of two stars which are probably not members. Using new parallaxes and available UCAC3 proper motions Weinberger et al. (2013) could not observe any convergence of the TWA group back in time. In the present work we used an updated database including the UCAC4 proper motions and new trigonometric parallaxes and observed a convergence for a restricted list of 16 stars. The data and the number of objects used to derive the age have a major impact on the result. Finally, the reality of the membership of the objects used for the backtracking is essential. Moreover, if some TWA groups of stars are not coeval then the task of finding an expansion age become even more complex. In this work we selected a subsample of probable coeval members of TWA and derived a traceback age for the association. This result will be considered in the next section via evaluation of individual star ages by the study of an HR diagram.

\section{HR diagram}

In the following, we construct an HR diagram of the TWA and determine the main physical parameters of individual stars using available photometric and spectroscopic information. Our analysis is obviously restricted to those stars marked with "Y" and "Y?" in Table 3 with known parallaxes and photometry. For a few systems that were resolved by high angular resolution photometric observations but with unresolved astrometry we assume the same parallax value for the various components.

Weinberger et al. (2013) derived the age of 29 TWA stars using their new parallax results and $H$ band photometry from the 2MASS catalog. No attempt was made in their work to correct the computed absolute magnitudes for extinction. In this paper we take into account the effect of stellar extinction due to circumstellar material that may be present in some association members (see Schneider et al. 2012a). We compute the visual extinction $A_{V}$ from the color excess in $(B-V),\left(V-I_{C}\right),(V-J)$, $(V-H)$ and $(V-K)$ following the procedure described by Pecaut et al. (2012). We also used $(J-K)$ from 2MASS photometry when optical colors were not available (see, e.g., Sect. 4.2 of Kenyon \& Hartmann 1995). Most stars in our sample exhibit low extinction, so we set the extinction to zero when derived estimates of $A_{V}$ yield a non-physical negative value that one might attribute to photometric errors. We adopt a total to selective extinction ratio of $R_{V}=3.1$ and took the weighted mean of all significant non-zero $A_{V}$ values as our final result. The formal uncertainties in our $A_{V}$ estimates that come from photometric errors are better than $0.1 \mathrm{mag}$. Our results are given in Table 5 . We use the value of $A_{H} / A_{V}=0.15$ from Cieza et al. (2005) to 
C. Ducourant et al.: The TW Hydrae association: trigonometric parallaxes and kinematic analysis

Table 5. Physical parameters derived for TWA stars with known distances.

\begin{tabular}{|c|c|c|c|c|c|c|c|c|c|c|}
\hline \multirow[b]{2}{*}{ Star } & \multirow[b]{2}{*}{ ST } & \multirow[b]{2}{*}{$\begin{array}{l}T_{\text {eff }} \\
(\mathrm{K})\end{array}$} & \multirow[b]{2}{*}{$\begin{array}{c}A_{V} \\
(\mathrm{mag})\end{array}$} & \multirow[b]{2}{*}{$\begin{array}{c}H_{\mathrm{abs}} \\
(\mathrm{mag})\end{array}$} & \multirow[b]{2}{*}{$\log \left(L / L_{\odot}\right)$} & \multicolumn{2}{|c|}{ Siess et al. (2000) } & \multicolumn{2}{|c|}{ Baraffe et al. (1998) } & \multirow[b]{2}{*}{ Ref. } \\
\hline & & & & & & $\log \left(M / M_{\odot}\right)$ & $\begin{array}{c}\log t \\
(t \text { in Myr })\end{array}$ & $\log \left(M / M_{\odot}\right)$ & $\begin{array}{c}\log t \\
(t \text { in Myr })\end{array}$ & \\
\hline TWA 1 & K8 & 3940 & 0.00 & $3.91 \pm 0.25$ & $-0.58 \pm 0.12$ & $-0.16 \pm 0.01$ & $6.90 \pm 0.19$ & $-0.10 \pm 0.03$ & $7.09 \pm 0.21$ & $1,2,3,4$ \\
\hline TWA 2A & M0.5 & 3704 & 0.33 & $4.22 \pm 0.44$ & $-0.75 \pm 0.20$ & $-0.34 \pm 0.01$ & $6.81 \pm 0.26$ & $-0.23 \pm 0.08$ & $7.02 \pm 0.29$ & $1,3,6,10$ \\
\hline TWA 2B & $\mathrm{M} 2$ & 3490 & 0.00 & $5.01 \pm 0.44$ & $-1.09 \pm 0.20$ & $-0.50 \pm 0.03$ & $6.91 \pm 0.24$ & $-0.39 \pm 0.06$ & $7.13 \pm 0.27$ & $1,3,5,6$ \\
\hline TWA 3A & M4 & 3160 & 0.00 & $4.79 \pm 0.13$ & $-1.03 \pm 0.07$ & $-0.75 \pm 0.02$ & $6.60 \pm 0.05$ & $-0.74 \pm 0.04$ & $6.38 \pm 0.07$ & $3,5,6$ \\
\hline TWA 3B & M4 & 3160 & 0.00 & $5.41 \pm 0.14$ & $-1.28 \pm 0.08$ & $-0.81 \pm 0.02$ & $6.78 \pm 0.06$ & $-0.81 \pm 0.03$ & $6.61 \pm 0.06$ & $3,5,6$ \\
\hline TWA 4A & K5 & 4140 & 0.00 & $3.31 \pm 0.24$ & $-0.31 \pm 0.12$ & $-0.06 \pm 0.01$ & $6.73 \pm 0.20$ & $-0.04 \pm 0.01$ & $6.81 \pm 0.22$ & $5,7,8$ \\
\hline TWA 4B & $\mathrm{K} 7, \mathrm{M} 1$ & 3970 & 0.00 & $3.32 \pm 0.24$ & $-0.34 \pm 0.12$ & $-0.16 \pm 0.01$ & $6.56 \pm 0.18$ & $-0.10 \pm 0.03$ & $6.70 \pm 0.20$ & $5,7,8$ \\
\hline TWA 5Aa & M2 & 3490 & 0.00 & $4.25 \pm 0.26$ & $-0.79 \pm 0.12$ & $-0.46 \pm 0.01$ & $6.55 \pm 0.10$ & $-0.40 \pm 0.06$ & $6.70 \pm 0.17$ & $1,3,5,9$ \\
\hline TWA $5 \mathrm{Ab}$ & M2 & 3490 & 0.00 & $4.35 \pm 0.26$ & $-0.83 \pm 0.12$ & $-0.47 \pm 0.01$ & $6.60 \pm 0.14$ & $-0.39 \pm 0.05$ & $6.75 \pm$ & $1,3,5,9$ \\
\hline TWA 6 & M0 & 3770 & 0.00 & $4.05 \pm 0.64$ & $-0.67 \pm 0.28$ & $-0.29 \pm 0.01$ & $6.72 \pm 0.39$ & $-0.19 \pm 0.06$ & 7.00 & $1,3,4$ \\
\hline TWA 7 & M3 & 3360 & 0.00 & $4.44 \pm 0.16$ & $-0.87 \pm 0.07$ & $-0.56 \pm 0.01$ & $6.61 \pm 0.08$ & $-0.54 \pm 0.05$ & $6.55 \pm 0.09$ & $1,3,4$ \\
\hline TWA 8A & M3 & 3360 & 0.00 & $4.57 \pm 0.21$ & $-0.93 \pm 0.11$ & $-0.56 \pm 0.02$ & $6.63 \pm 0.12$ & $-0.55 \pm 0.05$ & $6.62 \pm 0.15$ & $1,3,10$ \\
\hline TWA 9A & K7 & 3970 & 0.00 & $4.60 \pm 0.27$ & $-0.86 \pm 0.14$ & $-0.16 \pm 0.03$ & $7.38 \pm 0.22$ & $-0.15 \pm 0.08$ & $7.58 \pm 0.21$ & $1,2,3,10$ \\
\hline TWA 9B & M3.5 & 3255 & 0.00 & $5.83 \pm 0.16$ & $-1.44 \pm 0.09$ & $-0.74 \pm 0.01$ & $7.02 \pm 0.09$ & $-0.72 \pm 0.03$ & $6.97 \pm 0.11$ & $1,3,10$ \\
\hline TWA 10 & $\mathrm{M} 2$ & 3490 & 0.36 & $4.47 \pm 0.14$ & $-0.88 \pm 0.07$ & $-0.47 \pm 0.01$ & $6.66 \pm 0.08$ & $-0.39 \pm 0.05$ & $6.82 \pm 0.11$ & $1,3,4$ \\
\hline TWA $11 \mathrm{~A}$ & $\mathrm{~A} 0$ & 9700 & 0.00 & $1.48 \pm 0.06$ & $1.40 \pm 0.03$ & $+0.35 \pm 0.01$ & $6.80 \pm 0.06$ & $\ldots \ldots \ldots \ldots \ldots . .$. & $\ldots \ldots \ldots \ldots \ldots \ldots$ & $2,4,5$ \\
\hline TWA 11B & M2 & 3490 & 0.00 & $4.30 \pm 0.14$ & $-0.81 \pm 0.08$ & $-0.46 \pm 0.01$ & $6.58 \pm 0.09$ & $-0.40 \pm 0.05$ & & 5,10 \\
\hline TWA $11 \mathrm{C}$ & M4.5 & 3001 & 0.00 & $5.03 \pm 0.08$ & $-1.13 \pm 0.04$ & $-0.95 \pm 0.01$ & $6.60 \pm 0.02$ & $-1.00 \pm 0.02$ & $6.18 \pm$ & 1,4 \\
\hline TWA 12 & $\mathrm{M} 2$ & 3490 & 0.27 & $4.23 \pm 0.24$ & $-0.78 \pm 0.11$ & $-0.46 \pm 0.01$ & $6.55 \pm 0.12$ & $-0.40 \pm 0.06$ & $6.69 \pm 0.15$ & $1,3,4$ \\
\hline TWA 13A & M1 & 3630 & 0.00 & $4.00 \pm 0.11$ & $-0.67 \pm 0.06$ & $-0.38 \pm 0.01$ & $6.54 \pm 0.08$ & $-0.27 \pm 0.05$ & $6.80 \pm 0.08$ & $1,3,4$ \\
\hline TWA 13B & M1 & 3630 & 0.31 & $3.76 \pm 0.11$ & $-0.57 \pm 0.06$ & $-0.38 \pm 0.01$ & $6.42 \pm 0.07$ & $-0.28 \pm 0.05$ & $6.67 \pm 0.09$ & $1,3,4$ \\
\hline TWA 14 & M0 & 3770 & 0.00 & $4.57 \pm 0.26$ & $-0.88 \pm 0.12$ & $-0.30 \pm 0.01$ & $7.02 \pm 0.19$ & $-0.21 \pm 0.05$ & $7.33 \pm 0.19$ & $4,5,11$ \\
\hline TWA $15 \mathrm{~A}$ & M1.5 & 3562 & 0.00 & $4.73 \pm 0.41$ & $-0.97 \pm 0.18$ & $-0.44 \pm 0.01$ & $6.84 \pm 0.22$ & $-0.32 \pm 0.06$ & $7.10 \pm 0.25$ & $4,5,12$ \\
\hline TWA 15B & $\mathrm{M} 2$ & 3490 & 0.00 & $4.49 \pm 0.41$ & $-0.88 \pm 0.18$ & $-0.48 \pm 0.02$ & $6.73 \pm 0.20$ & $-0.39 \pm 0.05$ & $6.83 \pm 0.26$ & $4,5,12$ \\
\hline TWA 16 & M2 & 3490 & 0.00 & $4.56 \pm 0.10$ & $-0.91 \pm 0.06$ & $-0.47 \pm 0.01$ & $6.70 \pm 0.06$ & $-0.39 \pm 0.05$ & $6.88 \pm 0.09$ & $1,4,13$ \\
\hline TWA 20 & M3 & 3360 & 0.00 & $5.01 \pm 0.13$ & $-1.10 \pm 0.07$ & $-0.59 \pm 0.01$ & $6.81 \pm 0.07$ & $-0.56 \pm 0.05$ & $6.86 \pm 0.10$ & 1,4 \\
\hline TWA 21 & K3 & 4550 & 0.00 & $3.84 \pm 0.16$ & $-0.43 \pm 0.08$ & $-0.04 \pm 0.03$ & $7.41 \pm 0.10$ & $-0.05 \pm 0.05$ & $7.40 \pm 0.12$ & 1,4 \\
\hline TWA 23 & M1 & 3630 & 0.00 & $5.35 \pm 0.19$ & $-1.21 \pm 0.09$ & $-0.41 \pm 0.01$ & $7.25 \pm 0.13$ & $-0.28 \pm 0.06$ & $7.59 \pm 0.14$ & 1,4 \\
\hline TWA 25 & K9 & 3880 & 0.33 & $3.79 \pm 0.15$ & $-0.55 \pm 0.08$ & $-0.21 \pm 0.01$ & $6.72 \pm 0.11$ & $-0.12 \pm 0.03$ & $6.98 \pm 0.12$ & $1,3,4$ \\
\hline
\end{tabular}

Notes. We provide for each star the spectral type, temperature, visual extinction, absolute magnitude from $\mathrm{H}$ flux, luminosity, mass, and age derived from the Siess et al. (2000) and Baraffe et al. (1998) models, and the sources of photometric/spectroscopic information. The photometry of TWA 2, TWA 3, TWA 4, TWA 5, TWA 8, TWA 9, and TWA 16 are corrected for binarity with the information provided in the corresponding references (last column). TWA 14, TWA 20, and TWA 23 are corrected for binarity assuming that the components have equal brightness (as done by Weinberger et al. 2013). Note that our age and mass estimates for TWA 11C derived from the Baraffe et al. (1998) tracks and isochrones are only indicative because of the grid limits of this model and the star position in the HR diagram (see Fig. 6).

References. (1) Pecaut \& Mamajek (2013); (2) Hipparcos (ESA 1997); (3) Torres et al. (2006); (4) 2MASS (Cutri et al. 2003); (5) Schneider et al. (2012a); (6) Brandeker et al. (2003); (7) Fernández et al. (2008); (8) Prato et al. (2001); (9) Konopacky et al. (2007); (10) Webb et al. (1999); (11) Messina et al. (2010); (12) Barrado Y Navascués (2006); (13) Zuckerman et al. (2001); (14) Teixeira et al. (2008); (15) Looper et al. (2010a).

compute $A_{H}$ for each star and correct the stellar luminosities (see below) from stellar extinction. The $A_{H}$ values typically ranged from 0 mag to 0.05 mag.

The stellar luminosities were derived from the $H$ flux to better compare our results with Weinberger et al. (2013). However, to ensure the quality of our results we built a control sample by recalculating the stellar luminosities (and the stellar parameters) from the 2MASS $J$ flux. Both procedures yield equivalent results (within 8\%). So for clarity of presentation we have chosen to present in Table 5 only the stellar luminosities that result from the $H$ flux. The luminosity error budget takes into account photometric errors and the parallax uncertainty. We used the intrinsic colors, temperatures, and bolometric corrections for 5-30 Myr pre-main-sequence stars as given in Table 6 of Pecaut \& Mamajek $(2013)^{3}$.

The HR diagram presented in Fig. 6 shows that most TWA members lie between the $3 \mathrm{Myr}$ and $10 \mathrm{Myr}$ isochrones. We

\footnotetext{
3 Because TWA 11A is an A0 star (see, e.g., Schneider et al. 2012a), we used the intrinsic colors for dwarf stars given in Table 4 of Pecaut \& Mamajek (2013) to compute its luminosity.
}

derive the masses and ages of individual stars based on their position in the HR diagram. The models available for pre-mainsequence stars below $20 \mathrm{Myr}$ are rather uncertain (Soderblom et al. 2013) so we decided to test two of the more commonly used evolutionary models. To do so, we use the grid of pre-mainsequence evolutionary tracks and isochrones computed by Siess et al. (2000) and Baraffe et al. (1998). We use the version of the Baraffe et al. models with helium abundance $Y=0.282$ and mixing length parameter of 1.9 , because of its success in confirming coevality of the components in young multiple systems as reported by White et al. (1999). The stellar parameters that result from this investigation are presented in Table 5. We note that the stellar ages of the two components of the binary stars listed in Table 5 agree with each other within their errors. The age and mass distributions of TWA moving group members are shown in Fig. 7.

We note from Fig. 7 and Table 5 that TWA 9A, TWA 21, and TWA 23 are older than other moving group members according to both models. We noticed that the kinematical distance of TWA 9A calculated from the spatial velocity of the association as described in section $4.3\left(\pi_{\text {kin }}=14.2 \pm 1.0\right.$ mas $)$ and 
Siess et al. (2000)

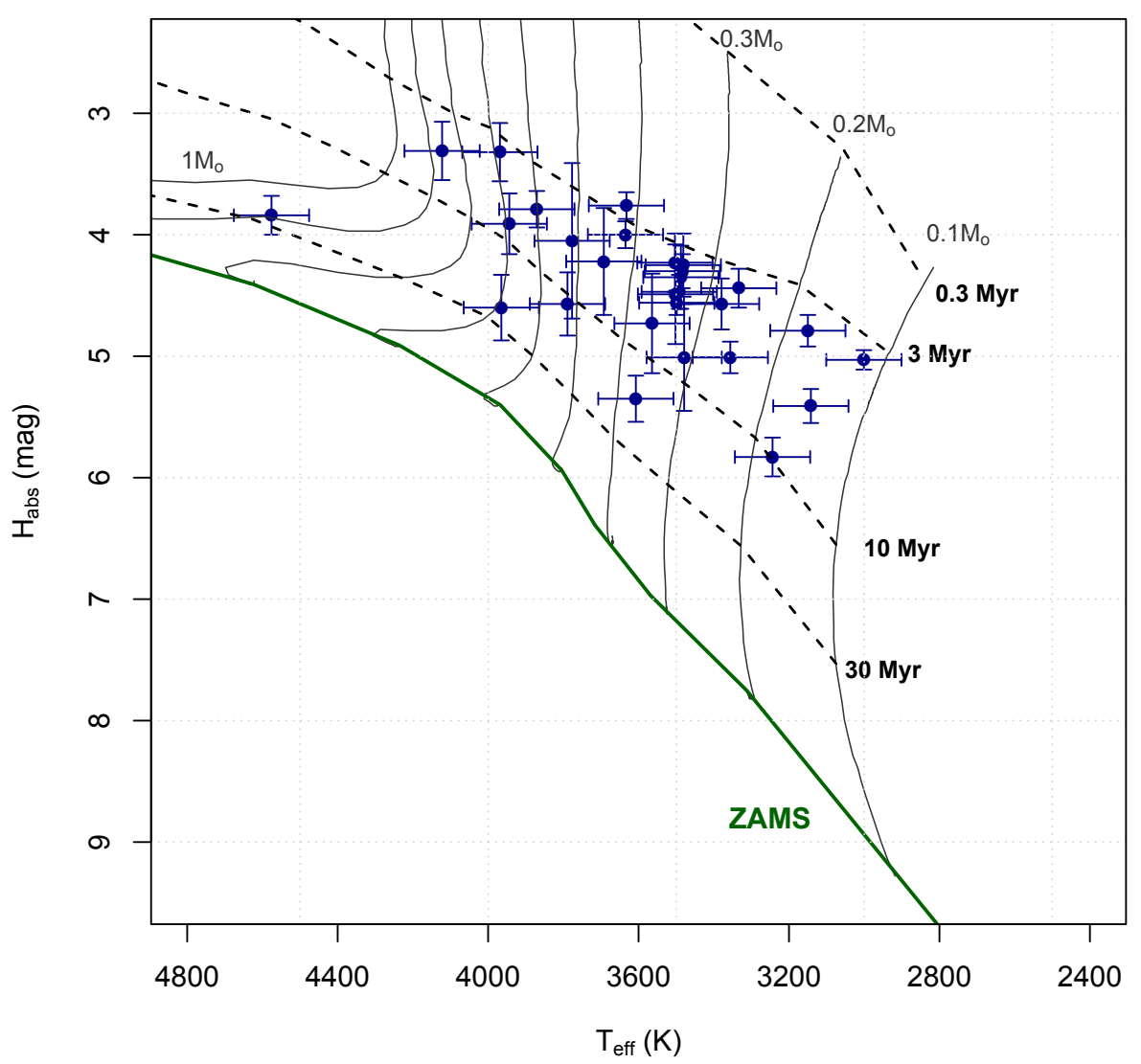

Baraffe et al. (1998)

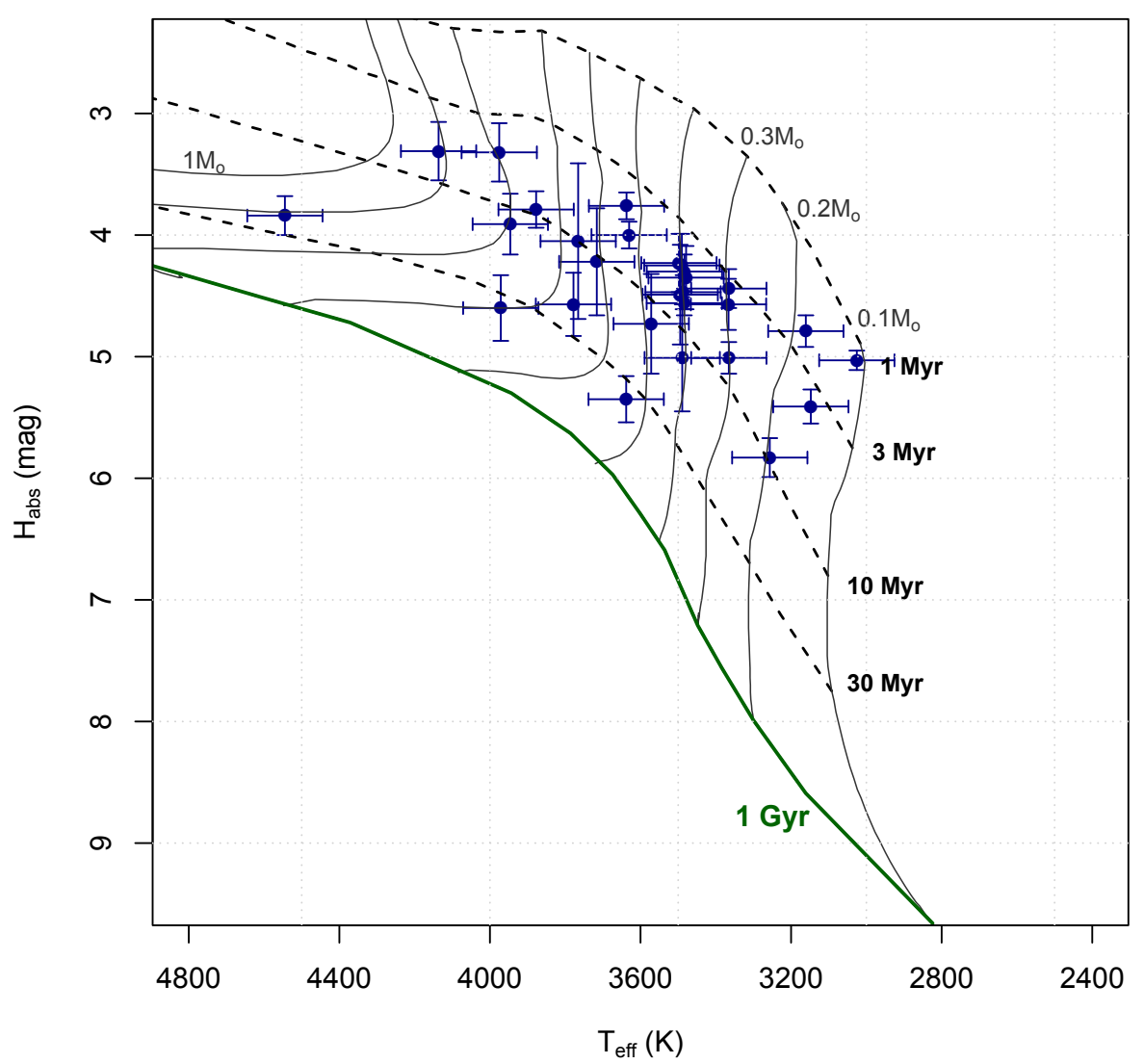

Fig. 6. HR diagram of the TWA stars listed in Table 5 with the grid of evolutionary models computed by Siess et al. (2000) with metallicity $Z=0.02$ (upper panel) and Baraffe et al. (1998) with $Y=0.282$ and mixing length parameter of 1.9 (lower panel). The solid and dashed lines are, respectively, evolutionary tracks from $0.1 M_{\odot}$ to $1 M_{\odot}$ with mass increment of $0.1 M_{\odot}$ and theoretical isochrones with the ages indicated in the figure. The green line indicates the zero-age main sequence (ZAMS) with the Siess et al. (2000) models, and the $1 \mathrm{Gyr}$ isochrone computed by Baraffe et al. (1998). We assume a $\pm 100 \mathrm{~K}$ uncertainty for all spectral types. 
C. Ducourant et al.: The TW Hydrae association: trigonometric parallaxes and kinematic analysis

Table 6. Comparison of age results inferred from the evolutionary models of Siess et al. (2000) and Baraffe et al. (1998).

\begin{tabular}{lcccccc}
\hline \hline & \multicolumn{3}{c}{ All stars } & \multicolumn{3}{c}{ Tracebackcore stars } \\
\hline & Nb Stars & $\begin{array}{c}\text { Mean age } \\
{[\mathrm{Myr}]}\end{array}$ & \multicolumn{2}{c}{$\begin{array}{c}\text { Median age } \\
{[\mathrm{Myr}]}\end{array}$} \\
\hline Siess et al. (2000) & 15 & $5.8 \pm 0.3$ & 5.4 & 11 & $5.9 \pm 0.4$ & 6.0 \\
Baraffe et al. (1998) & 17 & $8.2 \pm 0.7$ & 7.2 & 13 & $7.3 \pm 1.0$ & 6.3 \\
\hline
\end{tabular}

Notes. We provide the number of stars, mean age and median age derived from the evolutionary models of Siess et al. (2000) and Baraffe et al. (1998) after a $3 \sigma$ elimination for (i) all members of the association (marked with "Y" and "Y?" in Table 3), and (ii) the traceback-core stars listed in Sect. 5.2.
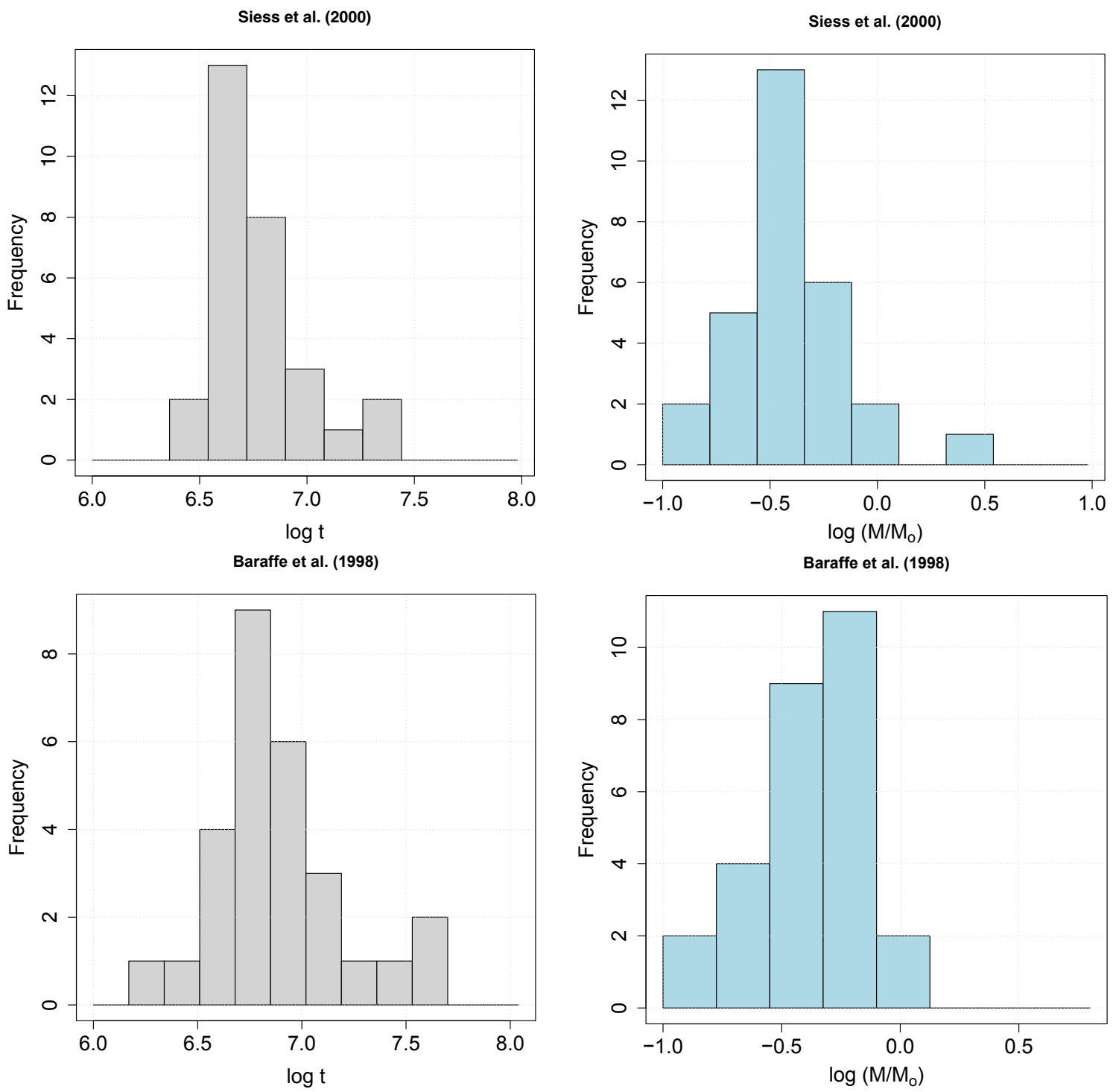

Fig. 7. Age (in $\log t$ ) and mass (in $\log M$ ) distributions for the TWA stars listed in Table 5 obtained with the grid of evolutionary models computed by Siess et al. 2000 (upper panels) and Baraffe et al. 1998 (lower panels). TWA 9A, TWA 21 and TWA 23 are the stars plotted near log $t=7.5$, and TWA 11A is the star plotted near $\log \left(M / M_{\odot}\right)=0.5$ in the upper panels. The Barrafe tracks do not cover such large masses.

its trigonometric distance $(\pi=21.4 \pm 2.5$ mas $)$ are very different. Weinberger et al. (2013) consider TWA 9A in their Sect. 4.2 and reject it as a member. Therefore, we strongly suspect that TWA 9A is not a TWA member or that its various trigonometric parallax measurements (this work, HIPPARcos) are false. Whatever, this star has been rejected from the traceback and is eliminated at a $3 \sigma$ rejection test when calculating the mean evolutionary age of TWA. Removing TWA 9A, TWA 21 and TWA 23 from our CP analysis in Sect. 4.1 has negligible effect on the $\mathrm{CP}$ position, thus they can still be regarded as kinematic members of the TWA. We also note from Table 5 that TWA 11A stands out on the mass distribution being more massive than other TWA members (see Fig. 7). This star does not appear in Fig. 6 because of the chosen range of temperature and luminosity.

The mean age for TWA stars derived in this work from the Baraffe et al. (1998) isochrones $(8.2 \pm 0.7 \mathrm{Myr})$ is consistent with the mean age of 9 Myr derived by Weinberger et al. (2013) 
in their analysis. However, the median age of 10.1 Myr reported in that analysis differs from the value of 7.2 Myr obtained in this work. The difference between these two age estimates can be due to some combination of a different sample of TWA stars, the effect of stellar extinction (not considered by Weinberger et al. 2013), and different ways of converting observed data (magnitudes, colors and spectral types) to theoretical values (effective temperature and luminosity). In Table 6 we present the mean and median age derived for each set of isochrones used in this work after a $3 \sigma$ elimination.

We note from Table 6 that the age estimates inferred from Siess et al. (2000) isochrones are systematically smaller than the results given by Baraffe et al. (1998). That the mean and median age results listed in Table 6 for each set of isochrones differ is not surprising given the different input assumptions and stellar physics in each model.

The mean of $8.2 \pm 0.7 \mathrm{Myr}$ inferred from the Baraffe et al. (1998) isochrones (after $3 \sigma$ elimination) is compatible with the dynamical traceback age within $1 \sigma$ of the computed errors. Furthermore, the mean age of $7.3 \pm 1.0 \mathrm{Myr}$ calculated using only the tracebackcore stars included in Table 5 is also fully consistent with the dynamical age and provides a more direct comparison of these age estimates. These results agree well with previous estimates using different strategies as summarized in Table 2 of Fernández et al. (2008). We conclude from this analysis that the dynamical traceback age derived in Sect. 5 is compatible with the isochronal age estimates derived from both evolutionary models used in this work, but it is in better agreement with the results inferred from the Baraffe et al. (1998) isochrones.

\section{Conclusions}

Based on astrometric and photometric observations performed with the ESO/NTT telescope we present trigonometric parallaxes, proper motions and photometry for 13 stars in the TW Hydrae association (TWA). This represents a gain of almost a factor of 3 when compared to the situation in the HIPPARcos era (only five stars with trigonometric parallaxes). The average precision of the parallaxes derived in the present study is 2.2 mas. Our proper motions are in good agreement with those given in UCAC4 and SPM4.

Using information provided in current astrometric catalogs and recent papers we set up an updated database for 34 previously proposed TWA members that allowed us to completely revisit the kinematics of the association.

Based on a convergent point search method we identify a moving group with 31 members and derive kinematic parallaxes for seven of these group members with unknown trigonometric parallaxes.

We derive the space motion for individual stars of the moving group and for 16 members trace motions back in time to find when these stars occupied a minimum volume. We derive a dynamical age of $7.5 \pm 0.7 \mathrm{Myr}$ for the association.

Using the parallaxes derived in this paper and published data we estimate mass and age for TWA moving group members from pre-main-sequence evolutionary models and find a mean age for the members as defined in Sect. 4.4 of $5.8 \pm 0.3 \mathrm{Myr}$ (Siess et al. 2000) and of 8.2 \pm 0.7 Myr (Baraffe et al. 1998).

To conclude, we show that the dynamical age of the association obtained via the traceback technique and the average ages derived from theoretical evolutionary models are compatible and that the Baraffe et al. (1998) mean age is in excellent agreement (within $1 \sigma$ ). We observe that the theoretical ages derived are dependent on multiple parameters and differ when applied to the same observational data while the dynamical age relies only on astrometric data and appears possibly more reliable.

Acknowledgements. We thank the referee for constructive comments that improved the paper. We also acknowledge partial financial support from the Brazilian organization FAPESP and CAPES, and the French organization COFECUB

\section{References}

Baraffe, I., Chabrier, G., Allard, F., \& Hauschildt, P. H. 1998, A\&A, 337, 403 Barrado Y Navascués, D. 2006, A\&A, 459, 511

Bergin, E. A., Cleeves, L. I., Gorti, U., et al. 2013, Nature, 493, 644

Bertout, C., \& Genova, F. 2006, A\&A, 460, 499

Binks, A. S., \& Jeffries, R. D. 2014, MNRAS, 438, L11

Bonnefoy, M., Chauvin, G., Dumas, C., et al. 2009, A\&A, 506, 799

Brandeker, A., Jayawardhana, R., \& Najita, J. 2003, AJ, 126, 2009

Chauvin, G., Lagrange, A.-M., Dumas, C., et al. 2004, A\&A, 425, L29

Chen, C. H., Mamajek, E. E., Bitner, M. A., et al. 2011, ApJ, 738, 122

Cieza, L. A., Kessler-Silacci, J. E., Jaffe, D. T., Harvey, P. M., \& Evans, II, N. J. 2005, ApJ, 635, 422

Cutri, R. M., Skrutskie, M. F., van Dyk, S., et al. 2003, VizieR Online Data Catalog: II/246

de Bruijne, J. H. J. 1999a, MNRAS, 306, 381

de Bruijne, J. H. J. 1999b, MNRAS, 310, 585

de la Reza, R., Torres, C. A. O., Quast, G., Castilho, B. V., \& Vieira, G. L. 1989, ApJ, 343, L61

de la Reza, R., Jilinski, E., \& Ortega, V. G. 2006, AJ, 131, 2609

Ducourant, C., Teixeira, R., Hambly, N. C., et al. 2007, A\&A, 470, 387

Ducourant, C., Teixeira, R., Chauvin, G., et al. 2008, A\&A, 477, L1

ESA 1997, VizieR Online Data Catalog: I/239

Fernández, D., Figueras, F., \& Torra, J. 2008, A\&A, 480, 735

Galli, P. A. B., Teixeira, R., Ducourant, C., Bertout, C., \& Benevides-Soares, P. 2012, A\&A, 538, A23

Girard, T. M., van Altena, W. F., Zacharias, N., et al. 2011, AJ, 142, 15

Gizis, J. E. 2002, ApJ, 575, 484

Gould, A. 2003, ArXiv Astrophysics

Høg, E., Fabricius, C., Makarov, V. V., et al. 2000, A\&A, 355, L27

Johnson, D. R. H., \& Soderblom, D. R. 1987, AJ, 93, 864

Kastner, J. H., Zuckerman, B., Weintraub, D. A., \& Forveille, T. 1997, Science, 277, 67

Kenyon, S. J., \& Hartmann, L. 1995, ApJS, 101, 117

Konopacky, Q. M., Ghez, A. M., Duchêne, G., McCabe, C., \& Macintosh, B. A. 2007, AJ, 133, 2008

Lawson, W. A., \& Crause, L. A. 2005, MNRAS, 357, 1399

Looper, D. L., Burgasser, A. J., Kirkpatrick, J. D., \& Swift, B. J. 2007, ApJ, 669, L97

Looper, D. L., Bochanski, J. J., Burgasser, A. J., et al. 2010a, AJ, 140, 1486

Looper, D. L., Mohanty, S., Bochanski, J. J., et al. 2010b, ApJ, 714, 45

Makarov, V. V. 2007, ApJS, 169, 105

Makarov, V. V., Gaume, R. A., \& Andrievsky, S. M. 2005, MNRAS, 362, 1109

Mamajek, E. E. 2005, ApJ, 634, 1385

Matthews, B. C., Kalas, P. G., \& Wyatt, M. C. 2007, ApJ, 663, 1103

Messina, S., Desidera, S., Turatto, M., Lanzafame, A. C., \& Guinan, E. F. 2010, A\&A, 520, A15

Mohanty, S., Jayawardhana, R., \& Barrado y Navascués, D. 2003, ApJ, 593, L109

Neuhäuser, R., Schmidt, T. O. B., Hambaryan, V. V., \& Vogt, N. 2010, A\&A, 516, A112

Ortega, V. G., de la Reza, R., Jilinski, E., \& Bazzanella, B. 2002, ApJ, 575, L75 Ortega, V. G., de la Reza, R., Jilinski, E., \& Bazzanella, B. 2004, ApJ, 609, 243

Pecaut, M. J., \& Mamajek, E. E. 2013, ApJS, 208, 9

Pecaut, M. J., Mamajek, E. E., \& Bubar, E. J. 2012, ApJ, 746, 154

Prato, L., Ghez, A. M., Piña, R. K., et al. 2001, ApJ, 549, 590

Reid, N. 2003, MNRAS, 342, 837

Riaz, B., \& Gizis, J. E. 2008, ApJ, 681, 1584

Riaz, B., \& Gizis, J. E. 2012, A\&A, 548, A54

Robin, A. C., Reylé, C., Derrière, S., \& Picaud, S. 2003, A\&A, 409, 523

Robin, A. C., Reylé, C., Derrière, S., \& Picaud, S. 2004, A\&A, 416, 157

Roeser, S., Demleitner, M., \& Schilbach, E. 2010, AJ, 139, 2440

Schneider, A., Melis, C., \& Song, I. 2012a, ApJ, 754, 39 
C. Ducourant et al.: The TW Hydrae association: trigonometric parallaxes and kinematic analysis

Schneider, A., Song, I., Melis, C., Zuckerman, B., \& Bessell, M. 2012b, ApJ, 757, 163

Shkolnik, E. L., Liu, M. C., Reid, I. N., Dupuy, T., \& Weinberger, A. J. 2011, ApJ, 727, 6

Siess, L., Dufour, E., \& Forestini, M. 2000, A\&A, 358, 593

Soderblom, D. R., Hillenbrand, L. A., Jeffries, R. D., Mamajek, E. E., \& Naylor, T. 2013 [arXiv: 1311.7024]

Song, I., Zuckerman, B., \& Bessell, M. S. 2003, ApJ, 599, 342

Song, I., Zuckerman, B., \& Bessell, M. S. 2012, AJ, 144, 8

Stetson, P. B. 1987, PASP, 99, 191

Teixeira, R., Ducourant, C., Chauvin, G., et al. 2008, A\&A, 489, 825

Teixeira, R., Ducourant, C., Chauvin, G., et al. 2009, A\&A, 503, 281
Torres, C. A. O., Quast, G. R., da Silva, L., et al. 2006, A\&A, 460, 695 Torres, C. A. O., Quast, G. R., Melo, C. H. F., \& Sterzik, M. F. 2008, in Young Nearby Loose Associations, ed. B. Reipurth, 757

Torres, G., Guenther, E. W., Marschall, L. A., et al. 2003, AJ, 125, 825 van Leeuwen, F. 2007, A\&A, 474, 653

Webb, R. A., Zuckerman, B., Platais, I., et al. 1999, ApJ, 512, L63

Weinberger, A. J., Anglada-Escudé, G., \& Boss, A. P. 2013, ApJ, 762, 118

White, R. J., Ghez, A. M., Reid, I. N., \& Schultz, G. 1999, ApJ, 520, 811

Zacharias, N., Finch, C. T., Girard, T. M., et al. 2013, AJ, 145, 44

Zuckerman, B., \& Song, I. 2004, ARA\&A, 42, 685

Zuckerman, B., Webb, R. A., Schwartz, M., \& Becklin, E. E. 2001, ApJ, 549, L233 